\title{
Simultaneous Determination of the Space-Dependent Source and the Initial Distribution in a Heat Equation by Regularizing Fourier Coefficients of the Given Measurements
}

\author{
Shufang Qiu (D), ${ }^{1,2}$ Wen Zhang, ${ }^{1,2}$ and Jianmei Peng ${ }^{1}$ \\ ${ }^{1}$ School of Science, East China University of Technology, Nanchang, Jiangxi 330013, China \\ ${ }^{2}$ Institute of Science and Engineering Computing, East China University of Technology, Nanchang 330013, China \\ Correspondence should be addressed to Shufang Qiu; shfqiu@ecit.cn
}

Received 15 January 2018; Accepted 20 May 2018; Published 2 July 2018

Academic Editor: Pavel Kurasov

Copyright (C) 2018 Shufang Qiu et al. This is an open access article distributed under the Creative Commons Attribution License, which permits unrestricted use, distribution, and reproduction in any medium, provided the original work is properly cited.

\begin{abstract}
We consider an inverse problem for simultaneously determining the space-dependent source and the initial distribution in heat conduction equation. First, we study the ill-posedness of the inverse problem. Then, we construct a regularization problem to approximate the originally inverse problem and obtain the regularization solutions with their stability and convergence results. Furthermore, convergence rates of the regularized solutions are presented under a prior and a posteriori strategies for selecting regularization parameters. Results of numerical examples show that the proposed regularization method is stable and effective for the considered inverse problem.
\end{abstract}

\section{Introduction}

In the past decades, various classes of inverse heat conduction equation problems have been studied by many scholars including recovery of the initial temperature [1-5], reconstruction of the heat source [6-12], and identification of thermal diffusion coefficients $[13,14]$. The inverse problems of heat equations such as the backward problems and the source reconstruction problems arise from various scientific and engineering fields, including heat conduction, hydrology, environmental controlling. It is worth noting that most of the existing literature considers recovery of only one unknown term or parameter. However, in many applications we hope to simultaneously reconstruct more than one unknown term from some overspecified conditions, which makes inverse problems very complicated. To the authors' knowledge, papers devoted to the simultaneous recovery problems are very limited. In [15], a numerical algorithm based on the fundamental solutions method is proposed to reconstruct the space-dependent heat source and the initial value simultaneously in an inverse heat conduction problem, which is transformed into a homogeneous backward problem and a
Dirichlet boundary value problem for Poisson's equation. In [16], an iterative algorithm is proposed for reconstructing both the space-dependent source term and the initial value based on solving a sequence of well-posed direct problems for the heat equation. In $[17,18]$, the unknown initial temperature and heat source are reconstructed simultaneously from the temperature data at the final time and at a fixed internal location over the time interval. In [19], the identification of the space-dependent heat source and the heat flux at the left endpoint is studied by the Tikhonov regularization method with generalized cross validation criterion for one-dimensional inverse heat conduction problem. In [20], the authors studied the inverse problem of reconstructing the time- and space-dependent heat source and the Robin boundary condition from the measured final data. In [21], the authors considered an inverse problem to simultaneously reconstruct the time- and space-dependent heat source and the initial temperature distribution and established the conditional stability and uniqueness of the inverse problem, which is solved by the variational regularization method. In [22], the inverse problem of simultaneous determination of the timedependent source term and the time-dependent coefficients 
in the heat equation is studied by the overspecified conditions of integral type. Recently, there has been a growing interest in inverse problems with fractional derivatives. In [23], the authors studied the inverse problem of the time-fractional diffusion equation in one-dimensional spatial space for determining the initial value and the heat flux on the boundary simultaneously and proved the uniqueness of the inverse problem by using the Laplace transform and the unique extension technique.

Motivated by the idea of $[3,5]$ for solving the backward problem of heat equation, we consider the inverse problem of heat equation to simultaneously determine the spacedependent source and the initial distribution from two final temperature measurements at two terminal times. This paper is organized as follows. The inverse problem is formulated in Section 2 with its ill-posedness. A regularization approximation problem is constructed to approximate the inverse problem, and regularized solutions are obtained with their stability and convergence rates in Section 3. Numerical examples are given in Section 4 to show the feasibility and validity of the proposed method. Finally, some conclusions are drawn in Section 5.

\section{Problem Formulation and Its Ill-Posedness}

2.1. Problem Formulation. Consider the initial-boundary problem of a nonhomogeneous heat conduction equation:

$$
\begin{aligned}
u_{t}-u_{x x} & =f(x), \quad(x, t) \in(0, \pi) \times[0, T], \\
u(0, t) & =u(\pi, t)=0, \quad t \in[0, T], \\
u(x, 0) & =\varphi(x), \quad x \in(0, \pi),
\end{aligned}
$$

where the initial distribution $\varphi(x)$ satisfies the compatibility condition: $\varphi(0)=\varphi(\pi)=0$. As we all know, the direct problem is solving problem (1) for yielding $u(x, t)$ when the source $f(x)$ and the initial distribution $\varphi(x)$ are known. However, the inverse problem considered in this paper is to reconstruct $f(x)$ and $\varphi(x)$ from problem (1) and two additional final measurements

$$
u\left(x, T_{1}\right)=g(x), \quad x \in(0, \pi),
$$

and

$$
u\left(x, T_{2}\right)=h(x), \quad x \in(0, \pi),
$$

where $T_{1}<T_{2}$.

2.2. Ill-Posedness. By the method of separation of variables, we obtain the solution to problem (1):

$$
u(x, t)=\sum_{k=1}^{\infty}\left(e^{-k^{2} t} \varphi_{k}+\frac{1-e^{-k^{2} t}}{k^{2}} f_{k}\right) \sin (k x),
$$

where $\varphi_{k}$ and $f_{k}$ are Fourier coefficients of $\varphi(x)$ and $f(x)$, respectively. And they are defined by

$$
\begin{aligned}
\varphi_{k} & =\frac{2}{\pi} \int_{0}^{\pi} \varphi(x) \sin (k x) d x \\
f_{k} & =\frac{2}{\pi} \int_{0}^{\pi} f(x) \sin (k x) d x
\end{aligned}
$$

From the Fourier expansions of $g(x)$ and $h(x)$ with respect to the eigenfunctions $\sin (k x)$, it follows that

$$
\begin{aligned}
& e^{-k^{2} T_{1}} \varphi_{k}+\frac{1-e^{-k^{2} T_{1}}}{k^{2}} f_{k}=g_{k}, \\
& e^{-k^{2} T_{2}} \varphi_{k}+\frac{1-e^{-k^{2} T_{2}}}{k^{2}} f_{k}=h_{k},
\end{aligned}
$$

where

$$
\begin{aligned}
& g_{k}=\frac{2}{\pi} \int_{0}^{\pi} g(x) \sin (k x) d x \\
& h_{k}=\frac{2}{\pi} \int_{0}^{\pi} h(x) \sin (k x) d x
\end{aligned}
$$

By simple calculations, from system (6) we obtain that

$$
\begin{aligned}
f_{k} & =\frac{k^{2}\left(h_{k}-g_{k} e^{-k^{2}\left(T_{2}-T_{1}\right)}\right)}{1-e^{-k^{2}\left(T_{2}-T_{1}\right)}}, \\
\varphi_{k} & =\frac{e^{k^{2} T_{1}}\left[h_{k}\left(1-e^{-k^{2} T_{1}}\right)-g_{k}\left(1-e^{-k^{2} T_{2}}\right)\right]}{e^{-k^{2}\left(T_{2}-T_{1}\right)}-1} .
\end{aligned}
$$

Thus, the solution to the inverse problem is obtained in the following form:

$$
\begin{aligned}
& f(x)=\sum_{k=1}^{\infty} \frac{k^{2}\left(h_{k}-g_{k} e^{-k^{2}\left(T_{2}-T_{1}\right)}\right)}{1-e^{-k^{2}\left(T_{2}-T_{1}\right)}} \sin (k x), \\
& \varphi(x)=\sum_{k=1}^{\infty} \frac{e^{k^{2} T_{1}}\left[h_{k}\left(1-e^{-k^{2} T_{1}}\right)-g_{k}\left(1-e^{-k^{2} T_{2}}\right)\right]}{e^{-k^{2}\left(T_{2}-T_{1}\right)}-1} \\
& \cdot \sin (k x) .
\end{aligned}
$$

Hence, the inverse problem has a unique solution $(f(x), \varphi(x))$ in $L^{2}(0, \pi)$ if and only if the Fourier coefficients of $g(x)$ and $h(x)$ satisfy that

$$
\sum_{k=1}^{\infty}\left|\frac{k^{2}\left(h_{k}-g_{k} e^{-k^{2}\left(T_{2}-T_{1}\right)}\right)}{1-e^{-k^{2}\left(T_{2}-T_{1}\right)}}\right|^{2}<\infty
$$

and

$$
\sum_{k=1}^{\infty}\left|\frac{e^{k^{2} T_{1}}\left[h_{k}\left(1-e^{-k^{2} T_{1}}\right)-g_{k}\left(1-e^{-k^{2} T_{2}}\right)\right]}{e^{-k^{2}\left(T_{2}-T_{1}\right)}-1}\right|^{2}<\infty .
$$


In other words, $g_{k}$ and $h_{k}$ must decay faster than $e^{-k^{2} T_{1}}$ as $k \rightarrow$ $+\infty$ for guaranteeing the existence of the inverse solution.

Unfortunately, the two additional measurements are always contaminated by noise in practical applications. In the context, we denote the contaminated measurements of $g(x)$ and $h(x)$ by $g^{\delta}(x)$ and $h^{\delta}(x)$, which satisfy that

$$
\begin{gathered}
\left\|g^{\delta}(x)-g(x)\right\| \leq \delta, \\
\left\|h^{\delta}(x)-h(x)\right\| \leq \delta .
\end{gathered}
$$

Here, $\|\cdot\|$ represents the $L^{2}$-norm on the interval $(0, \pi)$, and $\delta$ is the noise level. Although $g^{\delta}(x)$ and $h^{\delta}(x)$ belong to the space $L^{2}(0, \pi)$, the Fourier coefficients $\left\{g_{k}^{\delta}\right\}$ and $\left\{h_{k}^{\delta}\right\}$ no longer satisfy inequalities (11) and (12) since the random noise does not decay and will be dramatically amplified by $k^{2}$ and $e^{k^{2} T_{1}}$. Therefore, the inverse problem is ill-posed for determining the heat source and the initial distribution simultaneously in $L^{2}(0, \pi)$.

\section{Regularization Method}

First, we construct a regularization approximation problem for simultaneously determining the heat source term and the initial distribution:

$$
\begin{aligned}
& u_{t}^{\alpha}-u_{x x}^{\alpha}=f^{\alpha}(x), \quad(x, t) \in(0, \pi) \times[0, T], \\
& u^{\alpha}(0, t)=u^{\alpha}(\pi, t)=0, \quad t \in[0, T], \\
& u^{\alpha}(x, 0)=\varphi^{\alpha}(x), \quad x \in(0, \pi),
\end{aligned}
$$

and $u^{\alpha}(x, t)$ satisfies that

$$
\begin{array}{ll}
u^{\alpha}\left(x, T_{1}\right)=\sum_{k=1}^{\infty} \frac{e^{-k^{2} T_{1}}}{\alpha e^{k^{2}}+e^{-k^{2} T_{1}}} g_{k} \sin (k x), & \\
& x \in(0, \pi), \\
u^{\alpha}\left(x, T_{2}\right)=\sum_{k=1}^{\infty} \frac{e^{-k^{2} T_{2}}}{\alpha e^{k^{2}}+e^{-k^{2} T_{2}}} h_{k} \sin (k x), & x \in(0, \pi),
\end{array}
$$

where $\alpha \in(0,+\infty)$ is a regularization parameter. The superscript $\alpha$ is used to denote the dependency of the approximate solution on the regularization parameter. By using the method of separation of variables, we can easily derive the solution to the approximate problem (14):

$$
u^{\alpha}(x, t)=\sum_{k=1}^{\infty}\left(\frac{1-e^{-k^{2} t}}{k^{2}} f_{k}^{\alpha}+\varphi_{k}^{\alpha} e^{-k^{2} t}\right) \sin (k x),
$$

where

$$
\begin{aligned}
& f_{k}^{\alpha}=\frac{2}{\pi} \int_{0}^{\pi} f^{\alpha}(x) \sin (k x) d x, \\
& \varphi_{k}^{\alpha}=\frac{2}{\pi} \int_{0}^{\pi} \varphi^{\alpha}(x) \sin (k x) d x .
\end{aligned}
$$

From (15) and (16), we have

$$
\begin{aligned}
& \frac{1-e^{-k^{2} T_{1}}}{k^{2}} f_{k}^{\alpha}+\varphi_{k}^{\alpha} e^{-k^{2} T_{1}}=\frac{e^{-k^{2} T_{1}}}{\alpha e^{k^{2}}+e^{-k^{2} T_{1}}} g_{k}, \\
& \frac{1-e^{-k^{2} T_{2}}}{k^{2}} f_{k}^{\alpha}+\varphi_{k}^{\alpha} e^{-k^{2} T_{2}}=\frac{e^{-k^{2} T_{2}}}{\alpha e^{k^{2}}+e^{-k^{2} T_{2}}} h_{k} .
\end{aligned}
$$

Thus, the approximate solutions of the inverse problem are

$$
\begin{aligned}
& f^{\alpha}(x)=\sum_{k=1}^{\infty} \frac{k^{2}\left(\left(e^{-k^{2} T_{2}} /\left(\alpha e^{k^{2}}+e^{-k^{2} T_{2}}\right)\right) h_{k}-\left(e^{-k^{2} T_{1}} /\left(\alpha e^{k^{2}}+e^{-k^{2} T_{1}}\right)\right) g_{k} e^{-k^{2}\left(T_{2}-T_{1}\right)}\right)}{1-e^{-k^{2}\left(T_{2}-T_{1}\right)}} \sin (k x), \\
& \varphi^{\alpha}(x)=\sum_{k=1}^{\infty} \frac{e^{k^{2} T_{1}}\left[\left(e^{-k^{2} T_{2}} /\left(\alpha e^{k^{2}}+e^{-k^{2} T_{2}}\right)\right) h_{k}\left(1-e^{-k^{2} T_{1}}\right)-\left(e^{-k^{2} T_{1}} /\left(\alpha e^{k^{2}}+e^{-k^{2} T_{1}}\right)\right) g_{k}\left(1-e^{-k^{2} T_{2}}\right)\right]}{e^{-k^{2}\left(T_{2}-T_{1}\right)}-1} \sin (k x) .
\end{aligned}
$$

We firstly prove the posedness of problem (14)-(16). Since we have obtained the solution to problem (14)-(16), the existence and uniqueness of the solution are obvious. Therefore, we only need to prove the stability of the solution.

Before we prove the main theoretical results, we introduce a lemma.

Lemma 1. For $T>0,0<T_{1}<T_{2}$, and $\alpha \in(0,+\infty)$, the following inequalities are valid:

(a) $1 /\left(\alpha e^{\lambda}+e^{-T \lambda}\right) \leq T / \alpha\left(1+T(T / \alpha)^{1 /(1+T)}\right), \quad \lambda \in$ $(-\infty,+\infty)$. (b) $\lambda /\left(e^{\lambda T_{2}}-e^{\lambda T_{1}}\right) \leq 1 /\left(T_{2}-T_{1}\right), \quad \lambda \geq 1$.

(c) $e^{\lambda T_{2}} /\left(e^{\lambda T_{2}}-e^{\lambda T_{1}}\right) \leq e^{T_{2}} /\left(e^{T_{2}}-e^{T_{1}}\right), \lambda \geq 1$.

Proof. (a) We define the function $h(\lambda)$ by the formula

$$
h(\lambda)=\frac{1}{\alpha e^{\lambda}+e^{-T \lambda}}, \quad \lambda>0 .
$$

By simple computations, the function $h(\lambda)$ gets the maximum $h(\ln (T / \alpha) /(1+T))$ at the point $\lambda=\ln (T / \alpha) /(1+T)$; that is

$$
h(\lambda) \leq h\left(\frac{\ln (T / \alpha)}{1+T}\right) \leq \frac{T}{\alpha\left(1+T(T / \alpha)^{1 /(1+T)}\right)} .
$$


4

Advances in Mathematical Physics

(b)

$$
\begin{aligned}
\frac{\lambda}{e^{\lambda T_{2}}-e^{\lambda T_{1}}} & =\frac{\lambda}{e^{\lambda\left(T_{2}-T_{1}\right)}-1} e^{-\lambda T_{1}} \\
& \leq \frac{\lambda}{\left(\lambda\left(T_{2}-T_{1}\right)+1\right)-1} \leq \frac{1}{T_{2}-T_{1}} .
\end{aligned}
$$

(c) Set $F(\lambda)=e^{\lambda T_{2}} /\left(e^{\lambda T_{2}}-e^{\lambda T_{1}}\right)$. After a simple calculation we can say $F^{\prime}(\lambda)<0$, so the conclusion is true.

Theorem 2. Let $g(x), h(x), g^{\delta}(x), h^{\delta}(x) \in L^{2}(0, \pi)$ and satisfy (13), and then

$$
\begin{aligned}
& \left\|f^{\alpha}(x)-f^{\alpha, \delta}(x)\right\|_{L^{2}(0, \pi)} \\
& \quad \leq \frac{\sqrt{2 \pi}}{T_{2}-T_{1}} \frac{T_{2}}{\alpha\left(1+T_{2}\left(T_{2} / \alpha\right)^{1 /\left(1+T_{2}\right)}\right)} \delta,
\end{aligned}
$$

$$
\begin{aligned}
& f^{\alpha, \delta}(x)=\sum_{k=1}^{\infty} \frac{k^{2}\left(\left(e^{-k^{2} T_{2}} /\left(\alpha e^{k^{2}}+e^{-k^{2} T_{2}}\right)\right) h_{k}^{\delta}-\left(e^{-k^{2} T_{1}} /\left(\alpha e^{k^{2}}+e^{-k^{2} T_{1}}\right)\right) g_{k}^{\delta} e^{-k^{2}\left(T_{2}-T_{1}\right)}\right)}{1-e^{-k^{2}\left(T_{2}-T_{1}\right)}} \sin (k x), \\
& \varphi^{\alpha, \delta}(x)=\sum_{k=1}^{\infty} \frac{e^{k^{2} T_{1}}\left[\left(e^{-k^{2} T_{2}} /\left(\alpha e^{k^{2}}+e^{-k^{2} T_{2}}\right)\right) h_{k}^{\delta}\left(1-e^{-k^{2} T_{1}}\right)-\left(e^{-k^{2} T_{1}} /\left(\alpha e^{k^{2}}+e^{-k^{2} T_{1}}\right)\right) g_{k}^{\delta}\left(1-e^{-k^{2} T_{2}}\right)\right]}{e^{-k^{2}\left(T_{2}-T_{1}\right)}-1} \sin (k x),
\end{aligned}
$$

where

$$
\begin{aligned}
& g_{k}^{\delta}=\frac{2}{\pi} \int_{0}^{\pi} g^{\delta}(x) \sin (k x) d x, \\
& h_{k}^{\delta}=\frac{2}{\pi} \int_{0}^{\pi} h^{\delta}(x) \sin (k x) d x .
\end{aligned}
$$

From formulas (20) and (27) and Lemma 1, we can get

$$
\begin{aligned}
& \left\|f^{\alpha}(x)-f^{\alpha, \delta}(x)\right\|_{L^{2}(0, \pi)}^{2} \\
& =\mid \sum_{k=1}^{\infty} \frac{k^{2}}{1-e^{-k^{2}\left(T_{2}-T_{1}\right)}}\left[\frac{e^{-k^{2} T_{2}}}{\alpha e^{k^{2}}+e^{-k^{2} T_{2}}}\left(h_{k}-h_{k}^{\delta}\right)\right. \\
& \left.-\frac{e^{-k^{2} T_{1}}}{\alpha e^{k^{2}}+e^{-k^{2} T_{1}}} e^{-k^{2}\left(T_{2}-T_{1}\right)}\left(g_{k}-g_{k}^{\delta}\right)\right]\left.\sin (k x)\right|^{2} \\
& =\frac{\pi}{2} \sum_{k=1}^{\infty} \mid \frac{k^{2}}{e^{k^{2} T_{2}}-e^{k^{2} T_{1}}}\left[\frac{1}{\alpha e^{k^{2}}+e^{-k^{2} T_{2}}}\left(h_{k}-h_{k}^{\delta}\right)\right. \\
& \left.-\frac{1}{\alpha e^{k^{2}}+e^{-k^{2} T_{1}}}\left(g_{k}-g_{k}^{\delta}\right)\right]\left.\right|^{2} \leq \pi \sum_{k=1}^{\infty}\left[\frac{k^{2}}{e^{k^{2} T_{2}}-e^{k^{2} T_{1}}}\right. \\
& \left.\cdot \frac{1}{\alpha e^{k^{2}}+e^{-k^{2} T_{2}}}\right]^{2}\left[\left(h_{k}-h_{k}^{\delta}\right)^{2}+\left(g_{k}-g_{k}^{\delta}\right)^{2}\right]
\end{aligned}
$$

$$
\begin{aligned}
& \left\|\varphi^{\alpha}(x)-\varphi^{\alpha, \delta}(x)\right\|_{L^{2}(0, \pi)} \\
& \quad \leq \frac{\sqrt{2 \pi} e^{T_{2}}}{e^{T_{2}}-e^{T_{1}}} \frac{T_{2}}{\alpha\left(1+T_{2}\left(T_{2} / \alpha\right)^{1 /\left(1+T_{2}\right)}\right)} \delta ;
\end{aligned}
$$

ie., regularized solutions $f^{\alpha}(x)$ and $\varphi^{\alpha}(x)$ are consecutively dependent on the final value data $g(x)$ and $h(x)$, where $f^{\alpha, \delta}(x)$ and $\varphi^{\alpha, \delta}(x)$ are regularized solutions corresponding to $g^{\delta}(x)$ and $h^{\delta}(x)$, respectively.

Proof. From the perturbation data $g^{\delta}(x)$ and $h^{\delta}(x)$, by simple calculations we obtain$$
\leq \pi\left[\frac{1}{T_{2}-T_{1}} \frac{T_{2}}{\alpha\left(1+T_{2}\left(T_{2} / \alpha\right)^{1 /\left(1+T_{2}\right)}\right)}\right]^{2}(\| h
$$$$
\left.-h^{\delta}\left\|_{L^{2}(0, \pi)}^{2}+\right\| g-g^{\delta} \|_{L^{2}(0, \pi)}^{2}\right)
$$

i.e.,

$$
\begin{aligned}
& \left\|f^{\alpha}(x)-f^{\alpha, \delta}(x)\right\|_{L^{2}(0, \pi)} \\
& \quad \leq \frac{\sqrt{2 \pi}}{T_{2}-T_{1}} \frac{T_{2}}{\alpha\left(1+T_{2}\left(T_{2} / \alpha\right)^{1 /\left(1+T_{2}\right)}\right)} \delta .
\end{aligned}
$$

From formulas (21) and (28) and Lemma 1, we obtain

$$
\begin{aligned}
& \left\|\varphi^{\alpha}(x)-\varphi^{\alpha, \delta}(x)\right\|_{L^{2}(0, \pi)}^{2} \\
& =\mid \sum_{k=1}^{\infty} \frac{e^{k^{2} T_{1}}}{e^{-k^{2}\left(T_{2}-T_{1}\right)}-1}\left[\frac{e^{-k^{2} T_{2}}\left(1-e^{-k^{2} T_{1}}\right)}{\alpha e^{k^{2}}+e^{-k^{2} T_{2}}}\left(h_{k}-h_{k}^{\delta}\right)\right. \\
& \left.-\frac{e^{-k^{2} T_{1}}\left(1-e^{-k^{2} T_{2}}\right)}{\alpha e^{k^{2}}+e^{-k^{2} T_{1}}}\left(g_{k}-g_{k}^{\delta}\right)\right]\left.\sin (k x)\right|^{2}=\frac{\pi}{2} \\
& \cdot \sum_{k=1}^{\infty} \mid \frac{1}{e^{k^{2} T_{2}}-e^{k^{2} T_{1}}}\left[\frac{e^{k^{2} T_{1}}-1}{\alpha e^{k^{2}}+e^{-k^{2} T_{2}}}\left(h_{k}-h_{k}^{\delta}\right)\right.
\end{aligned}
$$


Advances in Mathematical Physics

5

$$
\begin{aligned}
& \left.-\frac{e^{k^{2} T_{2}}-1}{\alpha e^{k^{2}}+e^{-k^{2} T_{1}}}\left(g_{k}-g_{k}^{\delta}\right)\right]\left.\right|^{2} \leq \pi \sum_{k=1}^{\infty}\left[\frac{e^{k^{2} T_{2}}}{e^{k^{2} T_{2}}-e^{k^{2} T_{1}}}\right]^{2}\left[\left(h_{k}-h_{k}^{\delta}\right)^{2}+\left(g_{k}-g_{k}^{\delta}\right)^{2}\right] \\
& \left.\left.\cdot \frac{1}{\alpha e^{k^{2}}+e^{-k^{2} T_{2}}}\right]\right]^{2}(\| h \\
& \leq \pi\left[\frac{e^{T_{2}}}{e^{T_{2}}-e^{T_{1}}} \frac{T_{2}}{\alpha\left(1+T_{2}\left(T_{2} / \alpha\right)^{1 /\left(1+T_{2}\right)}\right)}\right]^{\delta} \\
& \left.-h^{\delta}\left\|_{L^{2}(0, \pi)}^{2}+\right\| g-g^{\delta} \|_{L^{2}(0, \pi)}^{2}\right) ;
\end{aligned}
$$

ie.,

$$
\begin{aligned}
& \left\|\varphi^{\alpha}(x)-\varphi^{\alpha, \delta}(x)\right\|_{L^{2}(0, \pi)} \\
& \quad \leq \frac{\sqrt{2 \pi} e^{T_{2}}}{e^{T_{2}}-e^{T_{1}}} \frac{T_{2}}{\alpha\left(1+T_{2}\left(T_{2} / \alpha\right)^{1 /\left(1+T_{2}\right)}\right)} \delta .
\end{aligned}
$$

Theorem 3. Let $g(x), h(x) \in L^{2}(0, \pi)$, and $\alpha \in(0, T)$. $f(x)$ and $\varphi(x)$ are the true solutions of the inverse problem (1). If there exists a constant A such that

$$
\sum_{k=1}^{\infty}\left[\left(e^{k^{2}\left(1+T_{2}\right)} h_{k}\right)^{2}+\left(e^{k^{2}\left(1+T_{1}\right)} g_{k}\right)^{2}\right] \leq A^{2},
$$

then

$$
\begin{aligned}
& \left\|f(x)-f^{\alpha}(x)\right\|_{L^{2}(0, \pi)} \\
& \quad \leq \frac{\sqrt{\pi}}{T_{2}-T_{1}} \frac{T_{2} A}{1+T_{2}\left(T_{2} / \alpha\right)^{1 /\left(1+T_{2}\right)}} . \\
& \left\|\varphi(x)-\varphi^{\alpha}(x)\right\|_{L^{2}(0, \pi)} \\
& \quad \leq \frac{\sqrt{\pi} e^{T_{2}}}{e^{T_{2}}-e^{T_{1}}} \frac{T_{2} A}{1+T_{2}\left(T_{2} / \alpha\right)^{1 /\left(1+T_{2}\right)}} .
\end{aligned}
$$

Proof. From formulas (9) and (20) and Lemma 1, we derive that

$$
\begin{aligned}
& \left\|f(x)-f^{\alpha}(x)\right\|_{L^{2}(0, \pi)}^{2} \\
& =\mid \sum_{k=1}^{\infty} \frac{k^{2}}{1-e^{-k^{2}\left(T_{2}-T_{1}\right)}}\left[\left(1-\frac{e^{-k^{2} T_{2}}}{\alpha e^{k^{2}}+e^{-k^{2} T_{2}}}\right) h_{k}\right. \\
& \left.-\left(1-\frac{e^{-k^{2} T_{1}}}{\alpha e^{k^{2}}+e^{-k^{2} T_{1}}}\right) e^{-k^{2}\left(T_{2}-T_{1}\right)} g_{k}\right]\left.\sin (k x)\right|^{2} \\
& =\frac{\pi}{2} \sum_{k=1}^{\infty} \mid \frac{k^{2}}{e^{k^{2} T_{2}}-e^{k^{2} T_{1}}}\left[\frac{\alpha}{\alpha e^{k^{2}}+e^{-k^{2} T_{2}} e^{k^{2}\left(1+T_{2}\right)} h_{k}}\right. \\
& -\frac{\alpha}{\left.\alpha e^{k^{2}}+e^{-k^{2} T_{1}} e^{k^{2}\left(1+T_{1}\right)} g_{k}\right]\left.\right|^{2} \leq \frac{\pi}{2} \sum_{k=1}^{\infty} \mid \frac{k^{2}}{e^{k^{2} T_{2}}-e^{k^{2} T_{1}}}}
\end{aligned}
$$

$$
\begin{aligned}
& \left.\cdot \frac{\alpha}{\alpha e^{k^{2}}+e^{-k^{2} T_{2}}}\left(e^{k^{2}\left(1+T_{2}\right)} h_{k}-e^{k^{2}\left(1+T_{1}\right)} g_{k}\right)\right|^{2} \leq \frac{\pi}{2} \\
& \cdot \sum_{k=1}^{\infty} \mid \frac{k^{2}}{e^{k^{2} T_{2}}-e^{k^{2} T_{1}}} \frac{\alpha}{\alpha e^{k^{2}}+e^{-k^{2} T_{2}}}\left(e^{k^{2}\left(1+T_{2}\right)} h_{k}\right. \\
& \left.-e^{k^{2}\left(1+T_{1}\right)} g_{k}\right)\left.\right|^{2} \leq \pi\left(\frac{1}{T_{2}-T_{1}}\right. \\
& \left.\cdot \frac{T_{2}}{1+T_{2}\left(T_{2} / \alpha\right)^{1 /\left(1+T_{2}\right)}}\right)^{2} A^{2} .
\end{aligned}
$$

i.e.,

$$
\begin{aligned}
& \left\|f(x)-f^{\alpha}(x)\right\|_{L^{2}(0, \pi)} \\
& \quad \leq \frac{\sqrt{\pi}}{T_{2}-T_{1}} \frac{T_{2} A}{1+T_{2}\left(T_{2} / \alpha\right)^{1 /\left(1+T_{2}\right)}} .
\end{aligned}
$$

From formulas (10) and (21) and Lemma 1, we can obtain

$$
\begin{aligned}
& \left\|\varphi(x)-\varphi^{\alpha}(x)\right\|_{L^{2}(0, \pi)}^{2} \\
& =\mid \sum_{k=1}^{\infty} \frac{e^{k^{2} T_{1}}}{e^{-k^{2}\left(T_{2}-T_{1}\right)}-1}\left[\frac{\alpha e^{k^{2}}\left(e^{-k^{2} T_{1}}-1\right)}{\alpha e^{k^{2}}+e^{-k^{2} T_{2}}} h_{k}\right. \\
& \left.+\frac{\alpha e^{k^{2}}\left(1-e^{-k^{2} T_{2}}\right)}{\alpha e^{k^{2}}+e^{-k^{2} T_{1}}} g_{k}\right]\left.\sin (k x)\right|^{2}=\frac{\pi}{2} \\
& \cdot \sum_{k=1}^{\infty} \mid \frac{e^{k^{2}\left(T_{1}+T_{2}\right)}}{e^{k^{2} T_{2}}-e^{k^{2} T_{1}}}\left[\frac{\alpha e^{k^{2}}\left(e^{-k^{2} T_{1}}-1\right)}{\alpha e^{k^{2}}+e^{-k^{2} T_{2}}} h_{k}\right. \\
& \left.+\frac{\alpha e^{k^{2}}\left(1-e^{-k^{2} T_{2}}\right)}{\alpha e^{k^{2}}+e^{-k^{2} T_{1}}} g_{k}\right]\left.\right|^{2} \leq \pi \sum_{k=1}^{\infty} \mid \frac{e^{k^{2} T_{2}}}{e^{k^{2} T_{2}}-e^{k^{2} T_{1}}}
\end{aligned}
$$

$\left.\frac{\alpha}{\alpha e^{k^{2}}+e^{-k^{2} T_{2}}}\right|^{2}\left[\left(e^{k^{2}\left(T_{1}+1\right)} h_{k}\right)^{2}+\left(e^{k^{2}\left(T_{1}+1\right)} g_{k}\right)^{2}\right]$

$$
\leq \pi\left(\frac{e^{T_{2}}}{e^{T_{2}}-e^{T_{1}}} \frac{T_{2}}{1+T_{2}\left(T_{2} / \alpha\right)^{1 /\left(1+T_{2}\right)}}\right)^{2} A^{2} ;
$$

ie.,

$$
\begin{aligned}
& \left\|\varphi(x)-\varphi^{\alpha}(x)\right\|_{L^{2}(0, \pi)} \\
& \quad \leq \frac{\sqrt{\pi} e^{T_{2}}}{e^{T_{2}}-e^{T_{1}}} \frac{T_{2} A}{1+T_{2}\left(T_{2} / \alpha\right)^{1 /\left(1+T_{2}\right)}} .
\end{aligned}
$$

Theorem 3 illustrates that $f^{\alpha}(x)$ and $\varphi^{\alpha}(x)$ are really regularized solutions, since $f^{\alpha}(x)$ and $\varphi^{\alpha}(x)$ approximate the true solutions $f(x)$ and $\varphi(x)$ as $\alpha \rightarrow 0$, respectively. 
Theorem 4. Under the conditions of Theorems 2 and 3, the choice $\alpha=\sqrt{2} \delta / A$ leads to asymptotically optimal estimates of the regularization solutions:

$$
\left\|f(x)-f^{\alpha, \delta}(x)\right\|_{L^{2}(0, \pi)}=O\left(\delta^{1 /\left(1+T_{2}\right)}\right)
$$

and

$$
\left\|\varphi(x)-\varphi^{\alpha, \delta}(x)\right\|_{L^{2}((0, \pi))}=O\left(\delta^{1 /\left(1+T_{2}\right)}\right) .
$$

Proof. From conclusions of Theorems 2 and 3, we have

$$
\begin{aligned}
\left\|f(x)-f^{\alpha, \delta}(x)\right\|_{L^{2}(0, \pi)} \\
\leq\left\|f(x)-f^{\alpha}(x)\right\|_{L^{2}(0, \pi)} \\
\quad+\left\|f^{\alpha}(x)-f^{\alpha, \delta}(x)\right\|_{L^{2}(0, \pi)} \\
\leq \frac{\sqrt{\pi}}{T_{2}-T_{1}} \frac{T_{2} A}{1+T_{2}\left(T_{2} / \alpha\right)^{1 /\left(1+T_{2}\right)}} \\
\quad+\frac{\sqrt{2 \pi}}{T_{2}-T_{1}} \frac{\delta T_{2}}{\alpha\left(1+T_{2}\left(T_{2} / \alpha\right)^{1 /\left(1+T_{2}\right)}\right)}
\end{aligned}
$$

and

$$
\begin{aligned}
\| \varphi(x) & -\varphi^{\alpha, \delta}(x) \|_{L^{2}(0, \pi)} \\
\leq & \left\|\varphi(x)-\varphi^{\alpha}(x)\right\|_{L^{2}(0, \pi)} \\
& +\left\|\varphi^{\alpha}(x)-\varphi^{\alpha, \delta}(x)\right\|_{L^{2}(0, \pi)} \\
\leq & \frac{\sqrt{\pi} e^{T_{2}}}{e^{T_{2}}-e^{T_{1}}} \frac{T_{2} A}{1+T_{2}\left(T_{2} / \alpha\right)^{1 /\left(1+T_{2}\right)}} \\
& +\frac{\delta T_{2}}{e^{T_{2}}-e^{T_{1}}} \frac{\delta}{\alpha\left(1+T_{2}\left(T_{2} / \alpha\right)^{1 /\left(1+T_{2}\right)}\right)} .
\end{aligned}
$$

From the above inequalities, the choice $\alpha=\sqrt{2} \delta / A$ leads to the asymptotically optimal estimates:

$$
\begin{gathered}
\left\|f(x)-f^{\alpha, \delta}(x)\right\|_{L^{2}(0, \pi)}=O\left(\delta^{1 /(1+T)}\right), \\
\left\|\varphi(x)-\varphi^{\alpha, \delta}(x)\right\|_{L^{2}(0, \pi)}=O\left(\delta^{1 /\left(1+T_{2}\right)}\right) .
\end{gathered}
$$

Theorem 4 gives a priori strategy for selecting regularization parameter. However, it is often undesirable to have the value of constant $A$ in practical applications. It is well known that regularization parameter plays a very important role in solving ill-posed problems, and the effectiveness of a regularization method depends strongly on the choice of the regularization parameter. Here, we adopt the Morozov discrepancy principle [24-26], a posteriori strategy, to select the regularization parameter; that is, selecting the regularization parameter $\alpha$ makes

$$
\begin{gathered}
\max \left\{\left\|u^{\alpha, \delta}\left(x, T_{1}\right)-g^{\delta}(x)\right\|^{2},\right. \\
\left.\left\|u^{\alpha, \delta}\left(x, T_{2}\right)-h^{\delta}(x)\right\|^{2}\right\}=C \delta^{2},
\end{gathered}
$$

where $C$ is a given constant. For regularization solutions $f^{\alpha, \delta}(x)$ and $\varphi^{\alpha, \delta}(x)$, the deviation equation (46) means that

$$
\begin{aligned}
& \max \left\{\sum_{k=1}^{\infty}\left(\frac{e^{-k^{2} T_{1}}}{\alpha e^{k^{2}}+e^{-k^{2} T_{1}}} g_{k}^{\delta}-g_{k}^{\delta}\right)^{2},\right. \\
& \left.\sum_{k=1}^{\infty}\left(\frac{e^{-k^{2} T_{2}}}{\alpha e^{k^{2}}+e^{-k^{2} T_{2}}} h_{k}^{\delta}-h_{k}^{\delta}\right)^{2}\right\}=C \delta^{2} .
\end{aligned}
$$

Theorem 5. Let $f(x)$ and $\varphi(x)$ be the true solutions of (1)-(3) with their Fourier coefficients $f_{k}$ and $g_{k}$, respectively. If there exist constants $P>0$ and $E$ such that

$$
\begin{aligned}
& \sum_{k=1}^{\infty}\left(e^{k^{2} T_{1}}\right)^{P} f_{k}^{2} \leq E, \\
& \sum_{k=1}^{\infty}\left(e^{k^{2} T_{1}}\right)^{P} \varphi_{k}^{2} \leq E,
\end{aligned}
$$

and $P T_{1}<2$, the a posteriori strategy (47) for selecting the regularization parameter leads to the following estimates:

$$
\begin{gathered}
\left\|f(x)-f^{\alpha, \delta}(x)\right\|_{L^{2}(0, \pi)}^{2}=O\left(\delta^{\min \{2 P /(P+2), 2\}}\right), \\
\left\|\varphi(x)-\varphi^{\alpha, \delta}(x)\right\|_{L^{2}(0, \pi)}^{2}=O\left(\delta^{\min \{2 P /(P+2), 2\}}\right),
\end{gathered}
$$

where $f^{\alpha, \delta}(x)$ and $\varphi^{\alpha, \delta}(x)$ are regularization solutions with respect to a regularization parameter selected by the a posteriori strategy (47).

Proof. (1) By careful computations and the Hölder inequality, we can get

$$
\begin{aligned}
& \left\|f(x)-f^{\alpha, \delta}(x)\right\|_{L^{2}(0, \pi)}^{2}=\sum_{k=1}^{\infty}\left|\frac{k^{2}}{1-e^{-k^{2}\left(T_{2}-T_{1}\right)}}\left[\left(h_{k}-\frac{e^{-k^{2} T_{2}}}{\alpha e^{k^{2}}+e^{-k^{2} T_{2}}} h_{k}^{\delta}\right)-e^{-k^{2}\left(T_{2}-T_{1}\right)}\left(g_{k}-\frac{e^{-k^{2} T_{1}}}{\alpha e^{k^{2}}+e^{-k^{2} T_{1}}} g_{k}^{\delta}\right)\right]\right|^{2} \\
& =\sum_{k=1}^{\infty}\left(\frac{k^{2}}{1-e^{-k^{2}\left(T_{2}-T_{1}\right)}}\right)^{2}\left[\left(h_{k}-\frac{e^{-k^{2} T_{2}}}{\alpha e^{k^{2}}+e^{-k^{2} T_{2}}} h_{k}^{\delta}\right)-e^{-k^{2}\left(T_{2}-T_{1}\right)}\left(g_{k}-\frac{e^{-k^{2} T_{1}}}{\alpha e^{k^{2}}+e^{-k^{2} T_{1}}} g_{k}^{\delta}\right)\right]^{4 /(P+2)}
\end{aligned}
$$


Advances in Mathematical Physics

7

$$
\begin{aligned}
& \cdot\left[\left(h_{k}-\frac{e^{-k^{2} T_{2}}}{\alpha e^{k^{2}}+e^{-k^{2} T_{2}}} h_{k}^{\delta}\right)-e^{-k^{2}\left(T_{2}-T_{1}\right)}\left(g_{k}-\frac{e^{-k^{2} T_{1}}}{\alpha e^{k^{2}}+e^{-k^{2} T_{1}}} g_{k}^{\delta}\right)\right]^{2-4 /(P+2)} \\
& \leq\left\{\sum_{k=1}^{\infty}\left[\left(\frac{k^{2}}{1-e^{-k^{2}\left(T_{2}-T_{1}\right)}}\right)^{2}\left[\left(h_{k}-\frac{e^{-k^{2} T_{2}}}{\alpha e^{k^{2}}+e^{-k^{2} T_{2}}} h_{k}^{\delta}\right)-e^{-k^{2}\left(T_{2}-T_{1}\right)}\left(g_{k}-\frac{e^{-k^{2} T_{1}}}{\alpha e^{k^{2}}+e^{-k^{2} T_{1}}} g_{k}^{\delta}\right)\right]^{4 /(P+2)}\right]^{(P+2) / 2}\right\}^{2 /(P+2)} \\
& \left\{\left[\sum_{k=1}^{\infty}\left[\left(h_{k}-\frac{e^{-k^{2} T_{2}}}{\alpha e^{k^{2}}+e^{-k^{2} T_{2}}} h_{k}^{\delta}\right)-e^{-k^{2}\left(T_{2}-T_{1}\right)}\left(g_{k}-\frac{e^{-k^{2} T_{1}}}{\alpha e^{k^{2}}+e^{-k^{2} T_{1}}} g_{k}^{\delta}\right)\right]^{2-4 /(P+2)}\right]^{(P+2) / P}\right\}^{P /(P+2)} \text {. }
\end{aligned}
$$

From (13), we can get

$$
\begin{aligned}
& \left\{\sum_{k=1}^{\infty}\left[\left(\frac{k^{2}}{1-e^{-k^{2}\left(T_{2}-T_{1}\right)}}\right)^{2}\left[\left(h_{k}-\frac{e^{-k^{2} T_{2}}}{\alpha e^{k^{2}}+e^{-k^{2} T_{2}}} h_{k}^{\delta}\right)-e^{-k^{2}\left(T_{2}-T_{1}\right)}\left(g_{k}-\frac{e^{-k^{2} T_{1}}}{\alpha e^{k^{2}}+e^{-k^{2} T_{1}}} g_{k}^{\delta}\right)\right]^{4 /(P+2)}\right]^{(P+2) / 2}\right\}^{2 /(P+2)} \\
& =\left\{\sum_{k=1}^{\infty}\left(\frac{k^{2}}{1-e^{-k^{2}\left(T_{2}-T_{1}\right)}}\right)^{P+2}\left[\left(h_{k}-\frac{e^{-k^{2} T_{2}}}{\alpha e^{k^{2}}+e^{-k^{2} T_{2}}} h_{k}^{\delta}\right)-e^{-k^{2}\left(T_{2}-T_{1}\right)}\left(g_{k}-\frac{e^{-k^{2} T_{1}}}{\alpha e^{k^{2}}+e^{-k^{2} T_{1}}} g_{k}^{\delta}\right)\right]^{2}\right\}^{2 /(P+2)} \\
& =\left\{\sum _ { k = 1 } ^ { \infty } ( \frac { k ^ { 2 } } { 1 - e ^ { - k ^ { 2 } ( T _ { 2 } - T _ { 1 } ) } } ) ^ { P + 2 } \left[\left(1-\frac{e^{-k^{2} T_{2}}}{\alpha e^{k^{2}}+e^{-k^{2} T_{2}}}\right) h_{k}+\frac{e^{-k^{2} T_{2}}}{\alpha e^{k^{2}}+e^{-k^{2} T_{2}}}\left(h_{k}-h_{k}^{\delta}\right)-e^{-k^{2}\left(T_{2}-T_{1}\right)} g_{k}\left(1-\frac{e^{-k^{2} T_{1}}}{\alpha e^{k^{2}}+e^{-k^{2} T_{1}}}\right)\right.\right. \\
& \left.\left.-e^{-k^{2}\left(T_{2}-T_{1}\right)} \frac{e^{-k^{2} T_{1}}}{\alpha e^{k^{2}}+e^{-k^{2} T_{1}}}\left(g_{k}-g_{k}^{\delta}\right)\right]^{2}\right\}^{2 /(P+2)} \leq\left\{2 \sum _ { k = 1 } ^ { \infty } ( \frac { k ^ { 2 } } { 1 - e ^ { - k ^ { 2 } ( T _ { 2 } - T _ { 1 } ) } } ) ^ { P + 2 } \left[\left(h_{k}-e^{-k^{2}\left(T_{2}-T_{1}\right)} g_{k}\right)^{2}\right.\right. \\
& \left.\left.+\left(\frac{e^{-k^{2} T_{2}}}{\alpha e^{k^{2}}+e^{-k^{2} T_{2}}}\left(h_{k}-h_{k}^{\delta}\right)+\frac{e^{-k^{2} T_{2}}}{\alpha e^{k^{2}}+e^{-k^{2} T_{1}}}\left(g_{k}-g_{k}^{\delta}\right)\right)^{2}\right]\right\}^{2 /(P+2)} \leq\left\{2 \sum_{k=1}^{\infty}\left(\frac{k^{2}}{1-e^{-k^{2}\left(T_{2}-T_{1}\right)}}\right)^{P} f_{k}^{2}+4 \sum_{k=1}^{\infty}\left(k^{2}\right)^{P+2}\right. \\
& \left.\cdot e^{-2 k^{2} T_{2}} \delta^{2}\right\}^{2 /(P+2)} \leq\left(2^{P+1} E+4 C_{1} \delta^{2}\right)^{2 /(P+2)}
\end{aligned}
$$

and

$$
\begin{aligned}
& \sum_{k=1}^{\infty}\left[\left(h_{k}-\frac{e^{-k^{2} T_{2}}}{\alpha e^{k^{2}}+e^{-k^{2} T_{2}}} h_{k}^{\delta}\right)-e^{-k^{2}\left(T_{2}-T_{1}\right)}\left(g_{k}-\frac{e^{-k^{2} T_{1}}}{\alpha e^{k^{2}}+e^{-k^{2} T_{1}}} g_{k}^{\delta}\right)\right]^{2-4 /(P+2)} \\
& \leq\left\{\left[\sum_{k=1}^{\infty}\left[\left(h_{k}-\frac{e^{-k^{2} T_{2}}}{\alpha e^{k^{2}}+e^{-k^{2} T_{2}}} h_{k}^{\delta}\right)-e^{-k^{2}\left(T_{2}-T_{1}\right)}\left(g_{k}-\frac{e^{-k^{2} T_{1}}}{\alpha e^{k^{2}}+e^{-k^{2} T_{1}}} g_{k}^{\delta}\right)\right]^{2-4 /(P+2)}\right]^{(P+2) / P}\right\}^{P /(P+2)} \\
& \leq\left\{2 \sum_{k=1}^{\infty}\left[\left(h_{k}-h_{k}^{\delta}\right)-\left(\frac{e^{-k^{2} T_{2}}}{\alpha e^{k^{2}}+e^{-k^{2} T_{2}}} h_{k}^{\delta}-h_{k}^{\delta}\right)\right]^{2}\right. \\
& \left.+2 \sum_{k=1}^{\infty}\left[e^{-k^{2}\left(T_{2}-T_{1}\right)}\left(\left(g_{k}-g_{k}^{\delta}\right)-\left(\frac{e^{-k^{2} T_{1}}}{\alpha e^{k^{2}}+e^{-k^{2} T_{1}}} g_{k}^{\delta}-g_{k}^{\delta}\right)\right)\right]^{2}\right\}^{P /(P+2)} \leq\left(8 C \delta^{2}+8 \delta^{2}\right)^{P /(P+2)}=C_{2} \delta^{2 P /(P+2)} \text {. }
\end{aligned}
$$


8

Advances in Mathematical Physics

In summary,

$$
\begin{aligned}
\| f & (x)-f^{\alpha, \delta}(x) \|_{L^{2}(0, \pi)}^{2} \\
& \leq\left(2^{P+1} E+4 C_{1} \delta^{2}\right)^{2 /(P+2)} C_{2} \delta^{2 P /(P+2)} \\
& \leq C_{3} E \delta^{2 P /(P+2)}+C_{4} \delta^{2}=O\left(\delta^{\min \{2 P /(P+2), 2\}}\right) .
\end{aligned}
$$

(2) Based on the Hölder inequality, we can similarly obtain that

(54)

$$
\begin{aligned}
& \left\|\varphi(x)-\varphi^{\alpha, \delta}(x)\right\|_{L^{2}(0, \pi)}^{2}=\sum_{k=1}^{\infty} \mid \frac{e^{k^{2} T_{1}}}{1-e^{-k^{2}\left(T_{2}-T_{1}\right)}}\left[\left(1-e^{-k^{2} T_{1}}\right)\left(\frac{e^{-k^{2} T_{2}}}{\alpha e^{k^{2}}+e^{-k^{2} T_{2}}} h_{k}^{\delta}-h_{k}\right)-\left(1-e^{-k^{2} T_{2}}\right)\right. \\
& \left.\cdot\left(\frac{e^{-k^{2} T_{1}}}{\alpha e^{k^{2}}+e^{-k^{2} T_{1}}} g_{k}^{\delta}-g_{k}\right)\right]\left.\right|^{2}=\sum_{k=1}^{\infty}\left(\frac{e^{k^{2} T_{1}}}{1-e^{-k^{2}\left(T_{2}-T_{1}\right)}}\right)^{2}\left[\left(1-e^{-k^{2} T_{1}}\right)\left(\frac{e^{-k^{2} T_{2}}}{\alpha e^{k^{2}}+e^{-k^{2} T_{2}}} h_{k}^{\delta}-h_{k}\right)-\left(1-e^{-k^{2} T_{2}}\right)\right. \\
& \left.\cdot\left(\frac{e^{-k^{2} T_{1}}}{\alpha e^{k^{2}}+e^{-k^{2} T_{1}}} g_{k}^{\delta}-g_{k}\right)\right]^{4 /(P+2)}\left[\left(1-e^{-k^{2} T_{1}}\right)\left(\frac{e^{-k^{2} T_{2}}}{\alpha e^{k^{2}}+e^{-k^{2} T_{2}}} h_{k}^{\delta}-h_{k}\right)-\left(1-e^{-k^{2} T_{2}}\right)\left(\frac{e^{-k^{2} T_{1}}}{\alpha e^{k^{2}}+e^{-k^{2} T_{1}}} g_{k}^{\delta}\right.\right. \\
& \left.\left.-g_{k}\right)\right]^{2-4 /(P+2)} \leq\left\{\sum _ { k = 1 } ^ { \infty } \left[\left(\frac{e^{k^{2} T_{1}}}{1-e^{-k^{2}\left(T_{2}-T_{1}\right)}}\right)^{2}\right.\right. \\
& \left.\left.\cdot\left[\left(1-e^{-k^{2} T_{1}}\right)\left(\frac{e^{-k^{2} T_{2}}}{\alpha e^{k^{2}}+e^{-k^{2} T_{2}}} h_{k}^{\delta}-h_{k}\right)-\left(1-e^{-k^{2} T_{2}}\right)\left(\frac{e^{-k^{2} T_{1}}}{\alpha e^{k^{2}}+e^{-k^{2} T_{1}}} g_{k}^{\delta}-g_{k}\right)\right]^{4 /(P+2)}\right]^{(P+2) / 2}\right\}^{2 /(P+2)} \\
& \left\{\left[\sum_{k=1}^{\infty}\left[\left(1-e^{-k^{2} T_{1}}\right)\left(\frac{e^{-k^{2} T_{2}}}{\alpha e^{k^{2}}+e^{-k^{2} T_{2}}} h_{k}^{\delta}-h_{k}\right)-\left(1-e^{-k^{2} T_{2}}\right)\left(\frac{e^{-k^{2} T_{1}}}{\alpha e^{k^{2}}+e^{-k^{2} T_{1}}} g_{k}^{\delta}-g_{k}\right)\right]^{2-4 /(P+2)}\right]^{2 /(P+2)}\right\}^{P /(P+2)}
\end{aligned}
$$

From inequalities (13), we get

$$
\begin{aligned}
& \left\{\sum _ { k = 1 } ^ { \infty } \left[\left(\frac{e^{k^{2} T_{1}}}{1-e^{-k^{2}\left(T_{2}-T_{1}\right)}}\right)^{2}\right.\right. \\
& \left.\left.\cdot\left[\left(1-e^{-k^{2} T_{1}}\right)\left(\frac{e^{-k^{2} T_{2}}}{\alpha e^{k^{2}}+e^{-k^{2} T_{2}}} h_{k}^{\delta}-h_{k}\right)-\left(1-e^{-k^{2} T_{2}}\right)\left(\frac{e^{-k^{2} T_{1}}}{\alpha e^{k^{2}}+e^{-k^{2} T_{1}}} g_{k}^{\delta}-g_{k}\right)\right]^{4 /(P+2)}\right]^{(P+2) / 2}\right\}^{2 /(P+2)} \\
& \leq 2\left\{\sum_{k=1}^{\infty}\left(\frac{e^{k^{2} T_{1}}}{1-e^{-k^{2}\left(T_{2}-T_{1}\right)}}\right)^{P+2}\left[\left(\frac{e^{-k^{2} T_{2}}}{\alpha e^{k^{2}}+e^{-k^{2} T_{2}}} h_{k}^{\delta}-h_{k}\right)^{2}+\left(\frac{e^{-k^{2} T_{1}}}{\alpha e^{k^{2}}+e^{-k^{2} T_{1}}} g_{k}^{\delta}-g_{k}\right)^{2}\right]\right\}^{2 /(P+2)} \\
& \leq 2\left\{\sum _ { k = 1 } ^ { \infty } ( \frac { e ^ { k ^ { 2 } T _ { 1 } } } { 1 - e ^ { - k ^ { 2 } ( T _ { 2 } - T _ { 1 } ) } } ) ^ { P + 2 } \left[\left(\frac{e^{-k^{2} T_{1}}}{\alpha e^{k^{2}}+e^{-k^{2} T_{1}}}\left(g_{k}^{\delta}-g_{k}\right)-\left(1-\frac{e^{-k^{2} T_{1}}}{\alpha e^{k^{2}}+e^{-k^{2} T_{1}}}\right) g_{k}\right)^{2}\right.\right. \\
& \left.\left.+\left(\frac{e^{-k^{2} T_{2}}}{\alpha e^{k^{2}}+e^{-k^{2} T_{2}}}\left(h_{k}^{\delta}-h_{k}\right)-\left(1-\frac{e^{-k^{2} T_{2}}}{\alpha e^{k^{2}}+e^{-k^{2} T_{2}}}\right) h_{k}\right)^{2}\right]\right\}^{2 /(P+2)} \leq 4\left\{\sum _ { k = 1 } ^ { \infty } ( \frac { e ^ { k ^ { 2 } T _ { 2 } } } { e ^ { k ^ { 2 } T _ { 2 } } - e ^ { k ^ { 2 } T _ { 1 } } } ) ^ { P + 2 } \left[\frac{e^{k^{2}\left(P T_{1}-2\right)}}{\alpha^{2}} \delta^{2}\right.\right.
\end{aligned}
$$


Advances in Mathematical Physics

9

$$
\begin{aligned}
& \left.\left.+e^{k^{2} T_{1}(P+2)} g_{k}^{2}+\frac{e^{k^{2}\left(P T_{1}-2\right)}}{\alpha^{2}} \delta^{2}+e^{k^{2} T_{1}(P+2)} h_{k}^{2}\right]\right\}^{2 /(P+2)} \leq 4\left\{\sum _ { k = 1 } ^ { \infty } ( \frac { e ^ { k ^ { 2 } T _ { 2 } } } { e ^ { k ^ { 2 } T _ { 2 } } - e ^ { k ^ { 2 } T _ { 1 } } } ) ^ { P + 2 } \left[2 \frac{e^{k^{2}\left(P T_{1}-2\right)}}{\alpha^{2}} \delta^{2}+e^{k^{2} T_{1}(P+2)} g_{k}^{2}\right.\right. \\
& \left.\left.+e^{k^{2} T_{1}(P+2)} h_{k}^{2}\right]\right\}^{2 /(P+2)} \leq 4\left\{( \frac { e ^ { k ^ { 2 } T _ { 2 } } } { e ^ { k ^ { 2 } T _ { 2 } } - e ^ { k ^ { 2 } T _ { 1 } } } ) ^ { P + 2 } \left[2 C_{1} \delta^{2}+e^{k^{2} T_{1}(P+2)}\left(e^{-k^{2} T_{1}} \varphi_{k}+\frac{1-e^{-k^{2} T_{1}}}{k^{2}} f_{k}\right)^{2}\right.\right. \\
& \left.\left.+e^{k^{2} T_{1}(P+2)}\left(e^{-k^{2} T_{2}} \varphi_{k}+\frac{1-e^{-k^{2} T_{2}}}{k^{2}} f_{k}\right)^{2}\right]\right\}^{2 /(P+2)} \leq 4\left\{\sum _ { k = 1 } ^ { \infty } ( \frac { e ^ { k ^ { 2 } T _ { 2 } } } { e ^ { k ^ { 2 } T _ { 2 } } - e ^ { k ^ { 2 } T _ { 1 } } } ) ^ { P + 2 } \left[2 C_{1} \delta^{2}\right.\right. \\
& \left.\left.+2 e^{k^{2} T_{1}(P+2)}\left(2 e^{-2 k^{2} T_{1}} \varphi_{k}^{2}+\frac{2 e^{2 k^{2}\left(T_{2}-T_{1}\right)}}{k^{4}} f_{k}^{2}\right)\right]\right\}^{2 /(P+2)} \leq 4\left\{\sum _ { k = 1 } ^ { \infty } ( \frac { e ^ { k ^ { 2 } T _ { 2 } } } { e ^ { k ^ { 2 } T _ { 2 } } - e ^ { k ^ { 2 } T _ { 1 } } } ) ^ { P + 2 } \left[2 C_{1} \delta^{2}+4 e^{k^{2} T_{1} P} \varphi_{k}^{2}+4\right.\right. \\
& \left.\left.\cdot \frac{e^{k^{2}\left(T_{1} P+2 T_{2}\right)}}{k^{4}} f_{k}^{2}\right]\right\}_{k=1}^{2 /(P+2)} \leq 4\left\{\sum_{k=1}^{\infty}\left(\frac{e^{T_{2}}}{e^{T_{2}}-e^{T_{1}}}\right)^{P+2}\left(2 C_{1} \delta^{2}+4 E+4 C_{2} E\right)\right\}^{2 /(P+2)} \leq 4\left(\frac{e^{T_{2}}}{e^{T_{2}}-e^{T_{1}}}\right)^{2}\left(2 C_{1} \delta^{2}\right. \\
& \left.+C_{3} E\right)^{2 /(P+2)}, \quad
\end{aligned}
$$

and

$$
\begin{aligned}
& \sum_{k=1}^{\infty}\left[\left(1-e^{-k^{2} T_{1}}\right)\left(\frac{e^{-k^{2} T_{2}}}{\alpha e^{k^{2}}+e^{-k^{2} T_{2}}} h_{k}^{\delta}-h_{k}\right)-\left(1-e^{-k^{2} T_{2}}\right)\left(\frac{e^{-k^{2} T_{1}}}{\alpha e^{k^{2}}+e^{-k^{2} T_{1}}} g_{k}^{\delta}-g_{k}\right)\right]^{2-4 /(P+2)} \\
& \leq\left\{\left[\sum_{k=1}^{\infty}\left[\left(1-e^{-k^{2} T_{1}}\right)\left(\frac{e^{-k^{2} T_{2}}}{\alpha e^{k^{2}}+e^{-k^{2} T_{2}}} h_{k}^{\delta}-h_{k}\right)-\left(1-e^{-k^{2} T_{2}}\right)\left(\frac{e^{-k^{2} T_{1}}}{\alpha e^{k^{2}}+e^{-k^{2} T_{1}}} g_{k}^{\delta}-g_{k}\right)\right]^{2-4 /(P+2)}\right]^{2 /(P+2)}\right\}^{P /(P+2)} \\
& \leq\left\{2 \sum_{k=1}^{\infty}\left[\left(1-e^{-k^{2} T_{1}}\right)\left(\left(\frac{e^{-k^{2} T_{2}}}{\alpha e^{k^{2}}+e^{-k^{2} T_{2}}} h_{k}^{\delta}-h_{k}^{\delta}\right)-\left(h_{k}-h_{k}^{\delta}\right)\right)\right]^{2}\right. \\
& \left.+2 \sum_{k=1}^{\infty}\left[\left(1-e^{-k^{2} T_{2}}\right)\left(\left(\frac{e^{-k^{2} T_{1}}}{\alpha e^{k^{2}}+e^{-k^{2} T_{1}}} g_{k}^{\delta}-g_{k}^{\delta}\right)-\left(g_{k}-g_{k}^{\delta}\right)\right)\right]^{2}\right\}^{P /(P+2)} \leq\left(8 C \delta^{2}+8 \delta^{2}\right)^{P /(P+2)}=C_{4} \delta^{2 P /(P+2)} .
\end{aligned}
$$

To sum up, we have

$$
\begin{aligned}
& \left\|\varphi(x)-\varphi^{\alpha, \delta}(x)\right\|_{L^{2}(0, \pi)}^{2} \\
& \leq 4\left(\frac{e^{T_{2}}}{e^{T_{2}}-e^{T_{1}}}\right)^{2}\left(2 C_{1} \delta^{2}+C_{3} E\right)^{2 /(P+2)} C_{4} \delta^{2 P /(P+2)} \\
& \leq C_{5} E \delta^{2 P /(P+2)}+C_{6} \delta^{2}=O\left(\delta^{\min \{2 P /(P+2), 2\}}\right) .
\end{aligned}
$$

4. Numerical Examples

In this section, we give two numerical examples to show effectiveness and stability of the proposed method: the first one has an analytical expression of the temperature concenration for the direct problem of heat conduction equation; the second one does not have an explicit solution of the direct problem, which must be solved by numerical methods such as the finite element method and the finite difference method.

In addition, the infinite summation in (27) and (28) must be truncated in practical computations. In the absence 
of confusion, we still denote the truncated solutions of regularization solutions by $f^{\alpha, \delta}(x)$ and $\varphi^{\alpha, \delta}(x)$; that is,

$$
\begin{aligned}
& f^{\alpha, \delta}(x)=\sum_{k=1}^{m} \frac{k^{2}\left(\left(e^{-k^{2} T_{2}} /\left(\alpha e^{k^{2}}+e^{-k^{2} T_{2}}\right)\right) h_{k}^{\delta}-\left(e^{-k^{2} T_{1}} /\left(\alpha e^{k^{2}}+e^{-k^{2} T_{1}}\right)\right) g_{k}^{\delta} e^{-k^{2}\left(T_{2}-T_{1}\right)}\right)}{1-e^{-k^{2}\left(T_{2}-T_{1}\right)}} \sin (k x), \\
& \varphi^{\alpha, \delta}(x)=\sum_{k=1}^{m} \frac{e^{k^{2} T_{1}}\left[\left(e^{-k^{2} T_{2}} /\left(\alpha e^{k^{2}}+e^{-k^{2} T_{2}}\right)\right) h_{k}^{\delta}\left(1-e^{-k^{2} T_{1}}\right)-\left(e^{-k^{2} T_{1}} /\left(\alpha e^{k^{2}}+e^{-k^{2} T_{1}}\right)\right) g_{k}^{\delta}\left(1-e^{-k^{2} T_{2}}\right)\right]}{e^{-k^{2}\left(T_{2}-T_{1}\right)}-1} \sin (k x) .
\end{aligned}
$$

Here, the Fourier coefficients $g_{k}^{\delta}$ and $h_{k}^{\delta}$ are computed by the trapezoid rule of numerical integral as follows:

$$
\begin{aligned}
g_{k}^{\delta} & =\frac{2}{\pi} \int_{0}^{\pi} g^{\delta}(x) \sin (k x) d x \\
& \approx \frac{2 h}{\pi} \sum_{i=1}^{n-1} g^{\delta}\left(x_{i}\right) \sin \left(k x_{i}\right)
\end{aligned}
$$

and

$$
\begin{aligned}
h_{k}^{\delta} & =\frac{2}{\pi} \int_{0}^{\pi} h^{\delta}(x) \sin (k x) d x \\
& \approx \frac{2 h}{\pi} \sum_{i=1}^{n-1} h^{\delta}\left(x_{i}\right) \sin \left(k x_{i}\right),
\end{aligned}
$$

where $g^{\delta}\left(x_{i}\right)=g\left(x_{i}\right)+\rho\left(2 \operatorname{rand}\left(x_{i}\right)-1\right) g\left(x_{i}\right), h^{\delta}\left(x_{i}\right)=$ $g\left(x_{i}\right)+\rho\left(2 \operatorname{rand}\left(x_{i}\right)-1\right) h\left(x_{i}\right), \rho$ is a relative error level, and rand is a random function in MATLAB that generates a uniformly distributed number over $(0,1)$. In all numerical experiments, we execute the Morozov discrepancy principle (46) for selecting regularization parameters as follows.

Let $r \in(0,1)$ be a fixed constant and $\alpha_{0}>0$ be an initial guess of the regularized parameter. Here, we consider regularization parameters as a geometric sequence form:

$$
\alpha_{k}=\alpha_{0} r^{k}, \quad k=0,1,2, \cdots
$$

The a posteriori strategy for selecting regularization parameters is to select the regularization parameter $\alpha_{k^{*}-1}$ so that in the first step $k^{*}$ has

$$
\begin{gathered}
\left\|u^{\alpha_{k^{*}, \delta}}\left(x, T_{1}\right)-g^{\delta}(x)\right\|_{L^{2}(0, \pi)}^{2} \leq C \delta^{2}, \\
\left\|u^{\alpha_{k^{*}, \delta}}\left(x, T_{2}\right)-h^{\delta}(x)\right\|_{L^{2}(0, \pi)}^{2} \leq C \delta^{2}
\end{gathered}
$$

where $C \geq 1$ is a constant and $u^{\alpha_{k}, \delta}\left(x, T_{1}\right)$ and $u^{\alpha_{k}, \delta}\left(x, T_{2}\right)$ are also the truncated solutions as the following forms:

$$
\begin{aligned}
& u^{\alpha_{k}, \delta}\left(x, T_{1}\right)=\sum_{k=1}^{m} \frac{e^{-k^{2} T_{1}}}{\alpha_{k} e^{k^{2}}+e^{-k^{2} T_{1}}} g_{k}^{\delta} \sin (k x), \\
& u^{\alpha_{k}, \delta}\left(x, T_{2}\right)=\sum_{k=1}^{m} \frac{e^{-k^{2} T_{2}}}{\alpha_{k} e^{k^{2}}+e^{-k^{2} T_{2}}} h_{k}^{\delta} \sin (k x) .
\end{aligned}
$$

We always take $n=100$ and $m=20$ and use two strategies for selecting regularization parameters in all numerical examples: (a) the a priori selection strategy given in Theorem 4 and (b) the a posteriori selection strategy 3.17 in which $\alpha_{0}=1, r=1 / 2$, and $C=1.0$. In order to compare the truncated regularization solutions with the true solutions, we introduce $E_{1}$ and $E_{2}$ representing the relative errors of regularization solutions and defined by

$$
\begin{aligned}
& E_{1}=\frac{\left(\sum_{i=0}^{n}\left|f^{\alpha, \delta}\left(x_{i}\right)-f\left(x_{i}\right)\right|^{2}\right)^{1 / 2}}{\left(\sum_{i=0}^{n}\left|f\left(x_{i}\right)\right|^{2}\right)^{1 / 2}}, \\
& E_{2}=\frac{\left(\sum_{i=0}^{n}\left|\varphi^{\alpha, \delta}\left(x_{i}\right)-\varphi\left(x_{i}\right)\right|^{2}\right)^{1 / 2}}{\left(\sum_{i=0}^{n}\left|\varphi\left(x_{i}\right)\right|^{2}\right)^{1 / 2}} .
\end{aligned}
$$

Example 1. Consider simultaneous determination of the source function and the initial distribution in the problem of heat conduction equation:

$$
\begin{aligned}
u_{t}-u_{x x} & =f(x), \quad(x, t) \in(0, \pi) \times[0,1], \\
u(0, t) & =u(\pi, t)=0, \quad(x, t) \in(0, \pi) \times[0,1], \\
u(x, 0) & =\varphi(x), \quad x \in(0, \pi), \\
u\left(x, T_{1}\right) & =g(x), \quad x \in(0, \pi), \\
u\left(x, T_{2}\right) & =h(x), \quad x \in(0, \pi),
\end{aligned}
$$

where $T_{1}=0.5, T_{2}=1, g(x)=\left(2-e^{-0.5}\right) \sin x$, and $h(x)=\left(2-e^{-1}\right) \sin x$. In this case, $f(x)=2 \sin x$ and $\varphi(x)=\sin x$. Numerical results of this example are shown in Table 1. Behaviors of the true solutions and the regularization solutions are shown in Figures 1 and 2.

Example 2. Consider simultaneous determination of the source function and the initial distribution in the problem of heat conduction equation:

$$
\begin{aligned}
u_{t}-u_{x x} & =f(x), \quad(x, t) \in(0, \pi) \times[0,1], \\
u(0, t) & =u(\pi, t)=0, \quad(x, t) \in(0, \pi) \times[0,1], \\
u(x, 0) & =\varphi(x), \quad x \in(0, \pi),
\end{aligned}
$$


TABLE 1: Numerical results of Example 1.

\begin{tabular}{lcccc}
\hline & $\delta$ & $\alpha$ & $E_{1}$ & $E_{2}$ \\
\hline \multirow{2}{*}{ a priori selection strategy } & 0.05 & $4.4678 \mathrm{e}-05$ & $2.9108 \mathrm{e}-03$ & $1.0786 \mathrm{e}-02$ \\
& 0.10 & $8.9357 \mathrm{e}-05$ & $5.1605 \mathrm{e}-03$ & $1.1633 \mathrm{e}-02$ \\
\hline \multirow{2}{*}{ a posteriori selection strategy } & 0.05 & $(1 / 2)^{12}$ & $2.3366 \mathrm{e}-03$ & $4.5157 \mathrm{e}-03$ \\
& 0.10 & $(1 / 2)^{11}$ & $3.8406 \mathrm{e}-03$ & $1.0433 \mathrm{e}-02$ \\
\hline
\end{tabular}
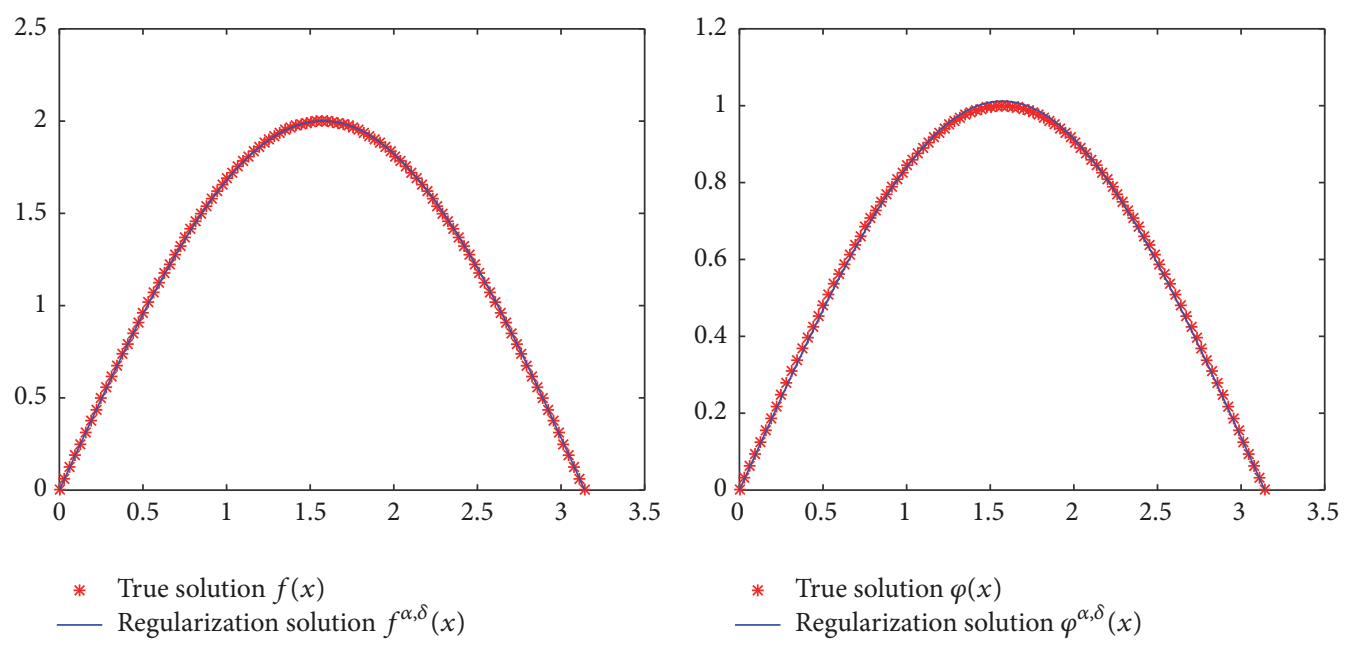

(a) Results with the a priori strategy for selecting regularization parameters
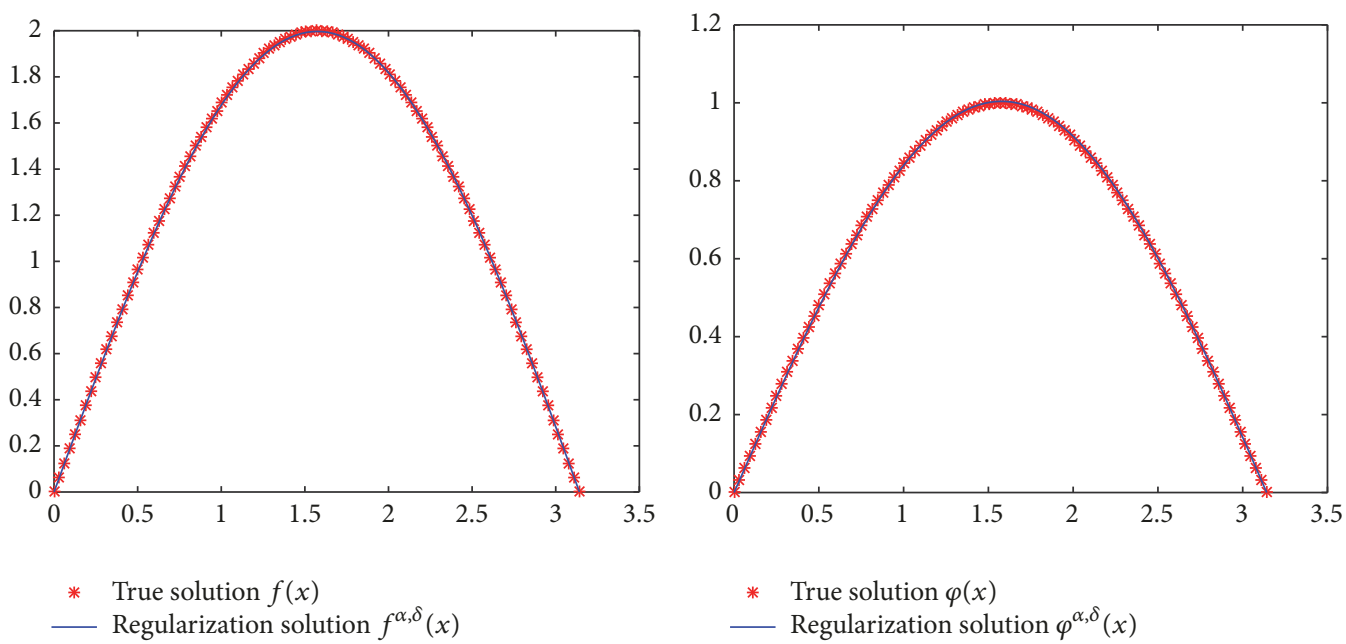

(b) Results with the a posteriori strategy for selecting regularization parameters

FIGURE 1: Behaviors of the true and the regularization solution when $\rho=0.05$.

where we take $f(x)=x(\pi-x)$ and $\varphi(x)=x \sin (x)$. Obviously, the direct problem does not have an analytical solution. So the temperature distribution functions $u\left(x, T_{1}\right)$ and $u\left(x, T_{2}\right)$ are obtained by using the inner function "pdepe" in MATLAB to solve the direct problem. Numerical results of this example are shown in Table 2. Behaviors of the true solutions and the regularization solutions are shown in Figures 3 and 4.

From Tables 1 and 2 and Figures 1-4, we can see that the proposed method is effective and stable for the noise data especially with large noise level. And the relative errors generated by using the a posteriori strategy for selecting regularization parameters are almost equivalent to the ones generated by using the a priori strategy for selecting regularization parameters, which also indicates that regularization parameters selected by the a posteriori strategy are reasonable for obtaining the meaningful inversion solutions.

Remark 6. It is well known that the backward heat conduction problem is a severely ill-posed problem. To show the influence of the final time values $T_{1}$ and $T_{2}$ on the numerical 

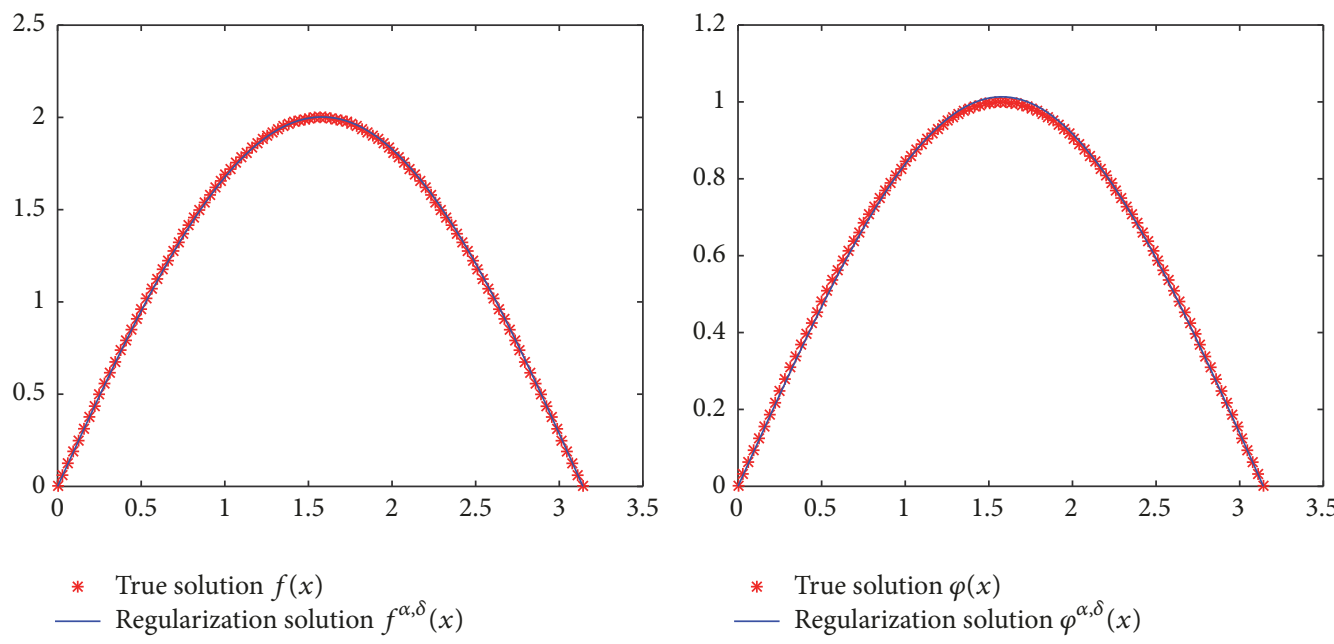

(a) Results with the a priori strategy for selecting regularization parameters
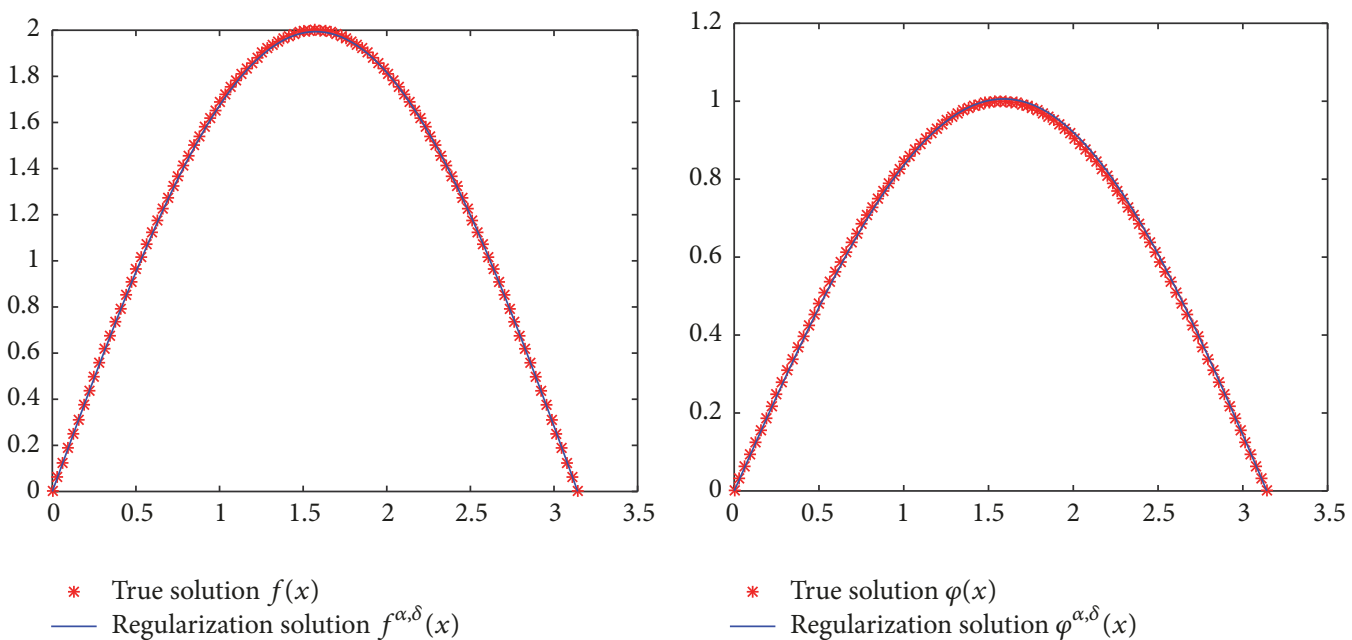

(b) Results with the a posteriori strategy for selecting regularization parameters

FIGURE 2: Behaviors of the true and the regularization solution when $\rho=0.10$.

TABLE 2: Numerical results of Example 2.

\begin{tabular}{lcccc}
\hline & $\delta$ & $\alpha$ & $E_{1}$ & $E_{2}$ \\
\hline \multirow{2}{*}{ a priori selection } & 0.05 & $5.9942 \mathrm{e}-05$ & $3.8019 \mathrm{e}-02$ & $2.8939 \mathrm{e}-02$ \\
& 0.10 & $1.1988 \mathrm{e}-04$ & $3.8028 \mathrm{e}-02$ & $2.8573 \mathrm{e}-02$ \\
\hline \multirow{2}{*}{ a posteriori selection } & 0.05 & $(1 / 2)^{12}$ & $3.8293 \mathrm{e}-02$ & $3.1005 \mathrm{e}-02$ \\
& 0.10 & $(1 / 2)^{10}$ & $3.9655 \mathrm{e}-02$ & $7.2344 \mathrm{e}-02$ \\
\hline
\end{tabular}

inversion results, we solve the inverse problem in Examples 1 and 2 by our proposed method with different large final time values and fixed values $n=200, m=20$, and $\delta=0.10$. Since the numerical results for Example 2 have the same behavior as Example 1, we only give the numerical results of Example 1 in Table 3, which show that the inversion results of the source term are better than that of the initial value with the increase of final time values $T_{1}$ and $T_{2}$. That is to say, the heat source can be reconstructed very well from the data measured at large final times, but the initial value is not available. It shows that the ill-posedness of the backward heat conduction problem is stronger than that of the inverse source problem.

\section{Conclusions and Discussion}

In this paper, we study an inverse problem for simultaneously determining the space-dependent source and the initial distribution in heat conduction equation. For obtaining stable solutions of the inverse problem, we construct a regularization problem to approximate the originally inverse problem. Stability and convergence of the regularization solutions are 

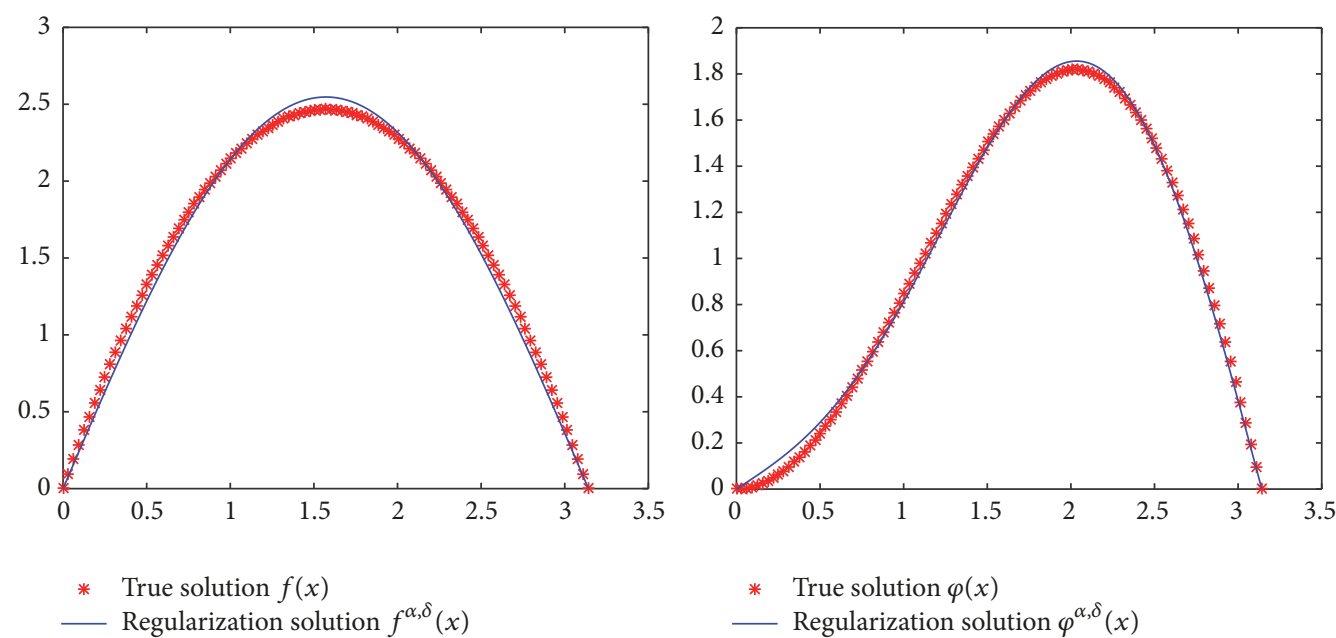

* True solution $f(x)$

— Regularization solution $f^{\alpha, \delta}(x)$

— Regularization solution $\varphi^{\alpha, \delta}(x)$

(a) Results with the a priori strategy for selecting regularization parameters
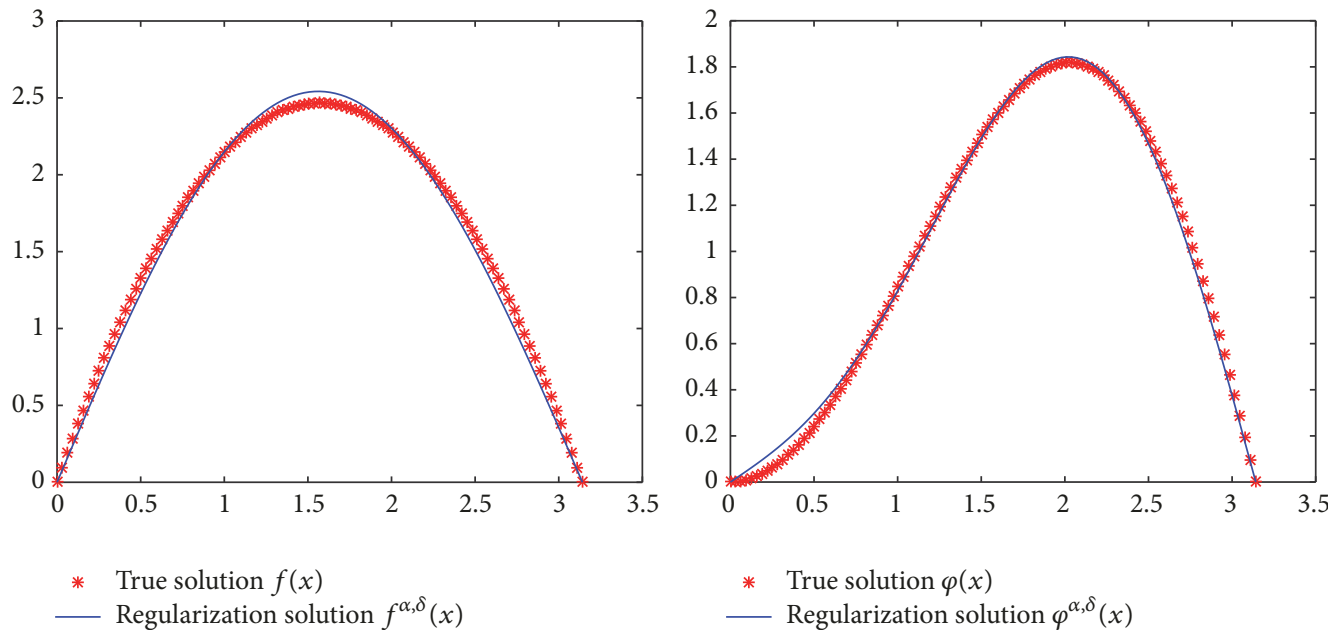

* True solution $f(x)$

- Regularization solution $f^{\alpha, \delta}(x)$

— Regularization solution $\varphi^{\alpha, \delta}(x)$

(b) Results with the a posteriori strategy for selecting regularization parameters

FIGURE 3: Behaviors of the true and the regularization solution when $\rho=0.05$.

TABle 3: Numerical results of Example 1 with different final time values.

\begin{tabular}{|c|c|c|c|c|c|}
\hline & $T_{1}$ & $T_{2}$ & $\alpha$ & $E_{1}$ & $E_{2}$ \\
\hline \multirow{7}{*}{ a priori selection strategy } & 1.0 & 1.5 & $7.3143 e-05$ & $1.7461 \mathrm{e}-03$ & $2.3633 e-03$ \\
\hline & 2.0 & 2.5 & $7.8949 \mathrm{e}-05$ & $4.4939 \mathrm{e}-03$ & $3.1173 \mathrm{e}-02$ \\
\hline & 3.0 & 3.5 & $8.1085 \mathrm{e}-05$ & $1.1924 \mathrm{e}-02$ & $2.6646 \mathrm{e}-01$ \\
\hline & 0.5 & 5.0 & $8.2050 \mathrm{e}-05$ & $3.2691 \mathrm{e}-02$ & $4.0627 \mathrm{e}-02$ \\
\hline & 1.0 & 5.0 & $8.2050 \mathrm{e}-05$ & $3.2922 \mathrm{e}-02$ & $1.0862 \mathrm{e}-01$ \\
\hline & 3.5 & 5.0 & $8.2050 \mathrm{e}-05$ & $3.9430 \mathrm{e}-02$ & $2.0275 \mathrm{e}+00$ \\
\hline & 5.0 & 6.0 & $8.2226 \mathrm{e}-05$ & $1.1243 \mathrm{e}-01$ & $2.3531 \mathrm{e}+01$ \\
\hline \multirow{7}{*}{ a posteriori selection strategy } & 1.0 & 1.5 & $(1 / 2)^{14}$ & $5262 \mathrm{e}-03$ & $2.2503 e-03$ \\
\hline & 2.0 & 2.5 & $(1 / 2)^{16}$ & $1.2060 \mathrm{e}-03$ & $3.4426 \mathrm{e}-02$ \\
\hline & 3.0 & 3.5 & $(1 / 2)^{17}$ & $1.5073 \mathrm{e}-03$ & $1.0573 e-01$ \\
\hline & 0.5 & 5.0 & $(1 / 2)^{19}$ & $1.1903 e-03$ & $2.4252 \mathrm{e}-02$ \\
\hline & 1.0 & 5.0 & $(1 / 2)^{19}$ & $1.1958 \mathrm{e}-03$ & $4.8508 \mathrm{e}-02$ \\
\hline & 3.5 & 5.0 & $(1 / 2)^{19}$ & $1.3532 \mathrm{e}-03$ & $4.2210 \mathrm{e}-01$ \\
\hline & 5.0 & 6.0 & $(1 / 2)^{21}$ & $1.1297 \mathrm{e}-03$ & $1.7172 \mathrm{e}+00$ \\
\hline
\end{tabular}



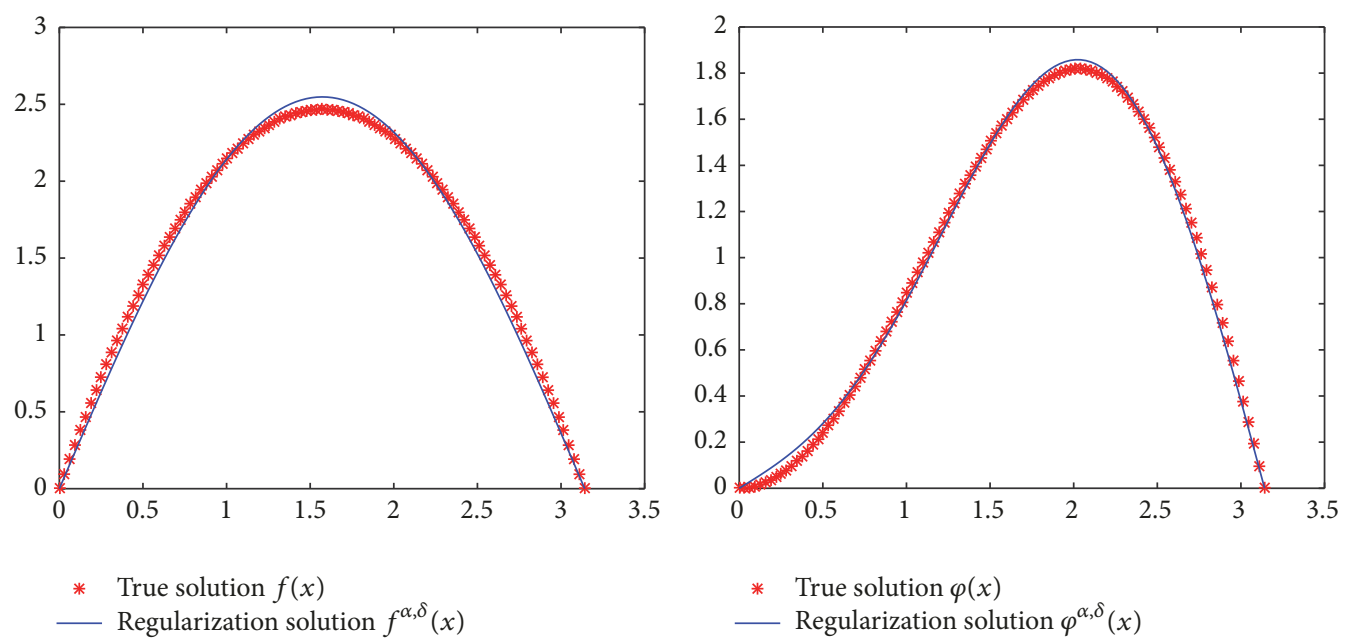

(a) Results with the a priori strategy for selecting regularization parameters
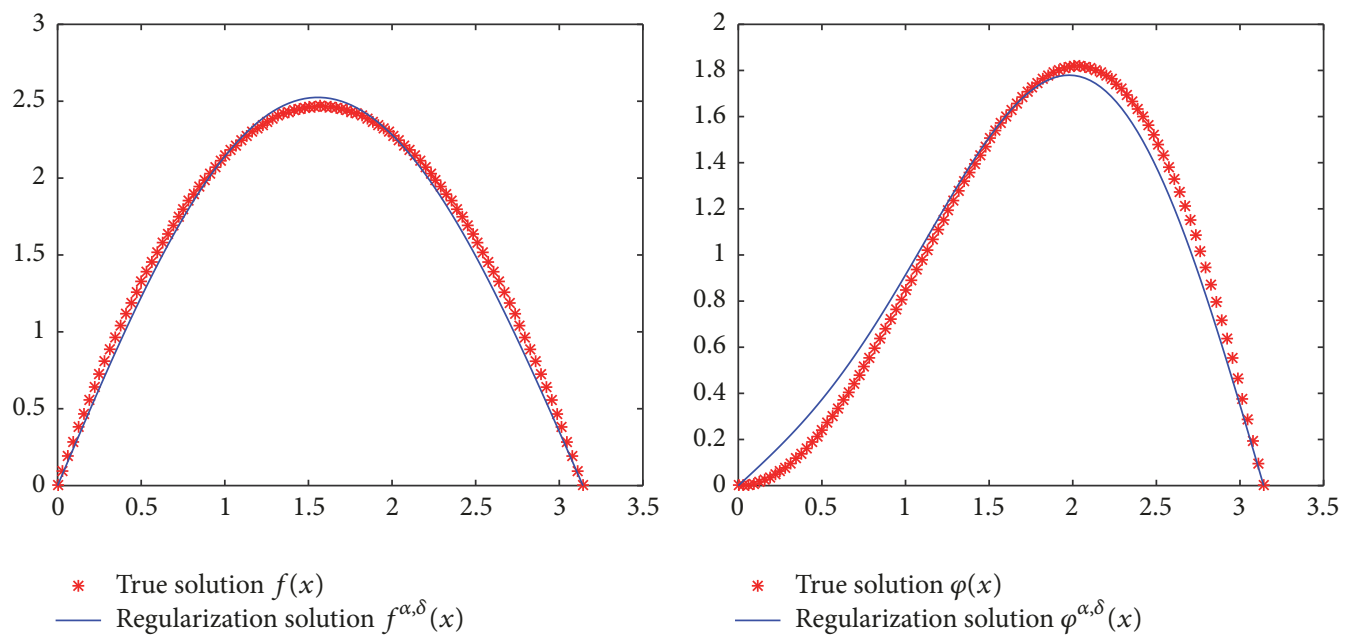

(b) Results with the a posteriori strategy for selecting regularization parameters

FIGURE 4: Behaviors of the true and the regularization solution when $\rho=0.10$.

proved, and convergence rates of the regularized solutions are also obtained under the a priori and a posteriori strategies for selecting regularization parameters, respectively. Numerical results show that the proposed regularization method is stable and effective for simultaneously determining the space-dependent source and the initial distribution from the measurements at suitable final time values.

\section{Data Availability}

The data generated during this study are available from the corresponding author upon request.

\section{Conflicts of Interest}

The authors declare that there are no conflicts of interest regarding the publication of this paper.

\section{Acknowledgments}

This work was supported by the National Natural Science Foundation of China [Grant nos. 11561003 and 11761007]; the Foundation of Academic and Technical Leaders Program for Major Subjects in Jiangxi Province [Grant no. 20172BCB22019]; the Natural Science Foundation of Jiangxi Province [Grant no. 20161BAB201034]; the Ground Project of Science and Technology of Jiangxi Universities [Grant no. KJLD14051].

\section{References}

[1] J. Cheng and J. J. Liu, "A quasi Tikhonov regularization for a two-dimensional backward heat problem by a fundamental solution," Inverse Problems, vol. 24, no. 6, Article ID 065012, pp. $1-18,2008$.

[2] D. N. Hào, N. Van, and D. Lesnic, "Regularization of parabolic equations backward in time by a non-local boundary value 
problem method,' IMA Journal of Applied Mathematics, vol. 75, no. 2, pp. 291-315, 2010.

[3] N. H. Tuan and D. D. Trong, "A new regularized method for two dimensional nonhomogeneous backward heat problem," Applied Mathematics and Computation, vol. 215, no. 3, pp. 873880, 2009.

[4] Y. C. Hon and T. Takeuchi, "Discretized Tikhonov regularization by reproducing kernel Hilbert space for backward heat conduction problem," Advances in Computational Mathematics, vol. 34, no. 2, pp. 167-183, 2011.

[5] N. H. Tuan, T. T. Binh, N. D. Minh, and T. T. Nghia, "An improved regularization method for initial inverse problem in 2-D heat equation," Applied Mathematical Modelling: Simulation and Computation for Engineering and Environmental Systems, vol. 39, no. 2, pp. 425-437, 2015.

[6] T. Johansson and D. Lesnic, "Determination of a spacewise dependent heat source," Journal of Computational and Applied Mathematics, vol. 209, no. 1, pp. 66-80, 2007.

[7] Z. Wang and J. Liu, "Identification of the pollution source from one-dimensional parabolic equation models," Applied Mathematics and Computation, vol. 219, no. 8, pp. 3403-3413, 2012.

[8] C. Sun, Q. Liu, and G. Li, “Conditional well-posedness for an inverse source problem in the diffusion equation using the variational adjoint method," Advances in Mathematical Physics, Art. ID 6801260, 6 pages, 2017.

[9] L. Yang, M. Dehghan, J. N. Yu et al., "Inverse problem of timedependent heat sources numerical reconstruction," Mathematics \& Computers in Simulation, vol. 81, no. 8, pp. 1656-1672, 2011.

[10] Z.-C. Deng, K. Qian, X.-B. Rao, L. Yang, and G.-W. Luo, "An inverse problem of identifying the source coefficient in a degenerate heat equation," Inverse Problems in Science and Engineering, vol. 23, no. 3, pp. 498-517, 2015.

[11] L. Yang, J.-N. Yu, G.-W. Luo, and Z.-C. Deng, "Numerical identification of source terms for a two dimensional heat conduction problem in polar coordinate system," Applied Mathematical Modelling: Simulation and Computation for Engineering and Environmental Systems, vol. 37, no. 3, pp. 939-957, 2013.

[12] L. Yang, J.-N. Yu, G.-W. Luo, and Z.-C. Deng, "Reconstruction of a space and time dependent heat source from finite measurement data," International Journal of Heat and Mass Transfer, vol. 55, no. 23-24, pp. 6573-6581, 2012.

[13] Q. Chen and J. J. Liu, "Solving an inverse parabolic problem by optimization from final measurement data," Journal of Computational and Applied Mathematics, vol. 193, no. 1, pp. 183203, 2006.

[14] Z.-C. Deng, Y.-C. Hon, and L. Yang, "An optimal control method for nonlinear inverse diffusion coefficient problem," Journal of Optimization Theory and Applications, vol. 160, no. 3, pp. 890-910, 2014.

[15] T. Wei and J. C. Wang, "Simultaneous determination for a space-dependent heat source and the initial data by the MFS," Engineering Analysis with Boundary Elements, vol. 36, no. 12, pp. 1848-1855, 2012.

[16] B. T. Johansson and D. Lesnic, "A procedure for determining a spacewise dependent heat source and the initial temperature," Applicable Analysis, vol. 87, no. 3, pp. 265-276, 2008.

[17] J. Wen, M. Yamamoto, and T. Wei, "Simultaneous determination of a time-dependent heat source and the initial temperature in an inverse heat conduction problem," Inverse Problems in Science and Engineering, vol. 21, no. 3, pp. 485-499, 2013.
[18] L. Yang, Z.-C. Deng, and Y.-C. Hon, "Simultaneous identification of unknown initial temperature and heat source," Dynamic Systems and Applications, vol. 25, no. 4, pp. 583-602, 2016.

[19] A. M. Shahrezaee and M. Rostamian, "Determination of a source term and boundary heat flux in an inverse heat equation," Journal of Information and Computing Science, vol. 8, no. 2, pp. 103-114, 2013.

[20] A. Hasanov, "Simultaneous determination of source terms in a linear parabolic problem from the final overdetermination: weak solution approach," Journal of Mathematical Analysis and Applications, vol. 330, no. 2, pp. 766-779, 2007.

[21] G.-H. Zheng and T. Wei, "Recovering the source and initial value simultaneously in a parabolic equation," Inverse Problems, vol. 30, no. 6, Article ID 065013, pp. 1-35, 2014.

[22] M. S. Hussein and D. Lesnic, "Simultaneous determination of time-dependent coefficients and heat source," International Journal for Computational Methods in Engineering Science and Mechanics, vol. 17, no. 5-6, pp. 401-411, 2016.

[23] J. J. Liu, M. Yamamoto, and L. Yan, "On the uniqueness and reconstruction for an inverse problem of the fractional diffusion process," Applied Numerical Mathematics, vol. 87, pp. 1-19, 2015.

[24] A. I. Prilepko, D. G. Orlovsky, and I. A. Vasin, Methods for Solving Inverse Problems in Mathematical Physics, vol. 231 of Monographs and Textbooks in Pure and Applied Mathematics, Marcel Dekker, New York, NY, USA, 2000.

[25] Z. Wang and J. Liu, "New model function methods for determining regularization parameters in linear inverse problems," Applied Numerical Mathematics, vol. 59, no. 10, pp. 2489-2506, 2009.

[26] Z.-w. Wang and D.-h. Xu, "On the linear model function method for choosing Tikhonov regularization parameters in linear ill-posed problems," Chinese Journal of Engineering Mathematics, vol. 30, no. 3, pp. 451-466, 2013. 


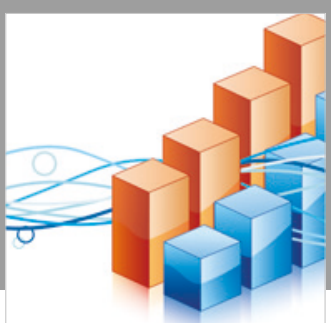

Advances in

Operations Research

\section{-n-m}
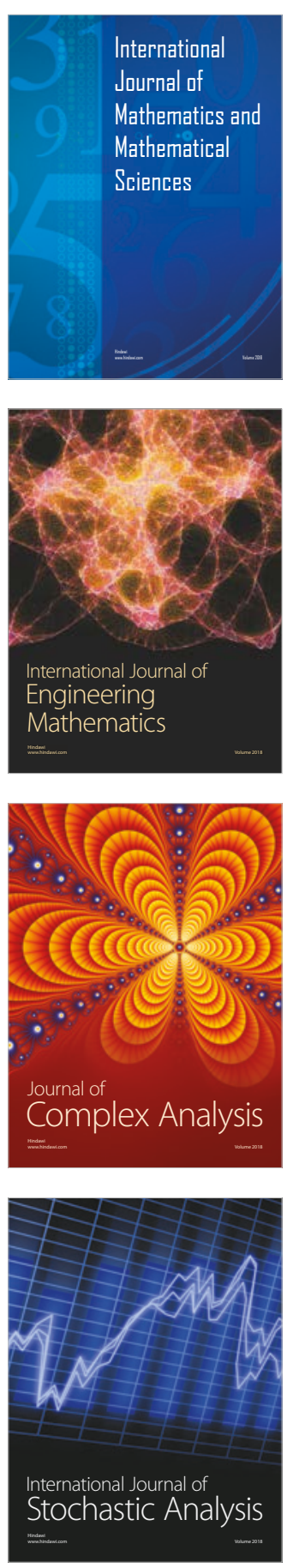
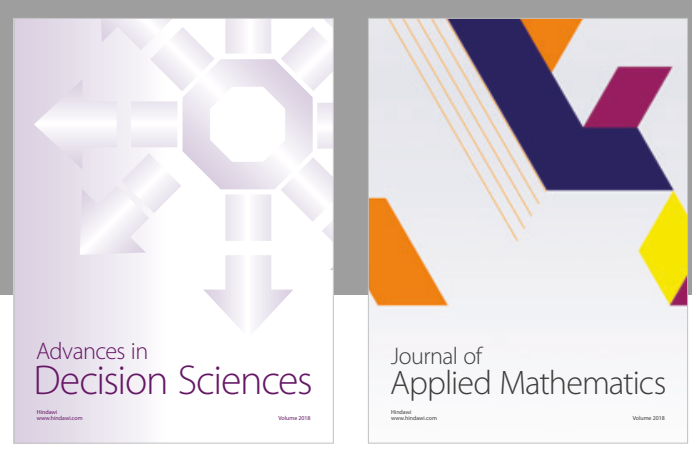

Journal of

Applied Mathematics
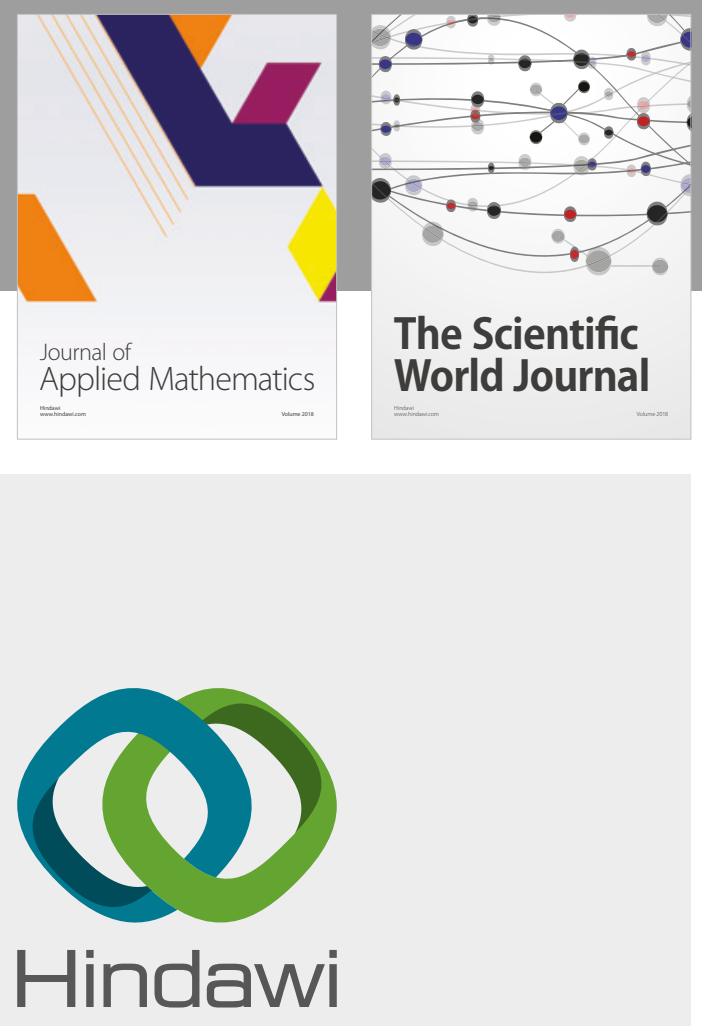

Submit your manuscripts at

www.hindawi.com

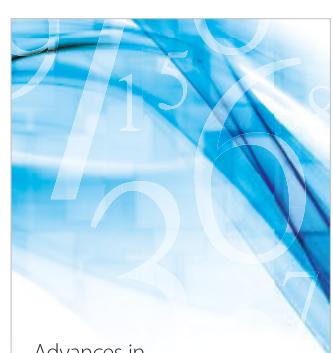

Advances in
Numerical Analysis
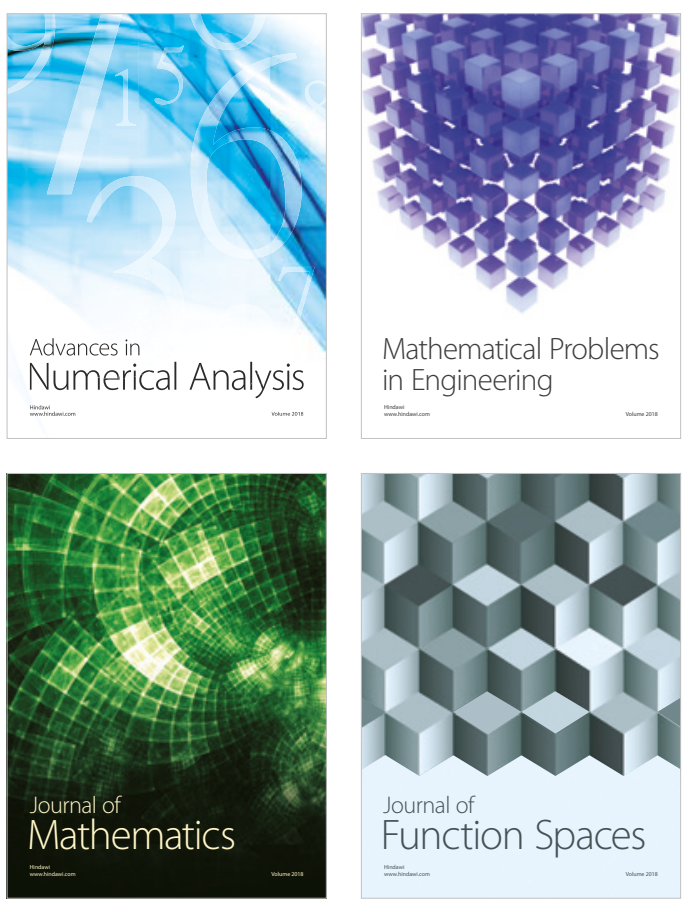

Mathematical Problems in Engineering

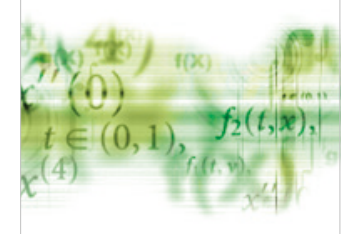

International Journal of

Differential Equations

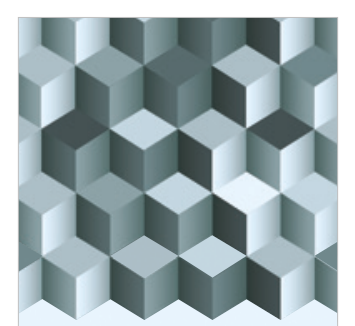

Journal of

Function Spaces

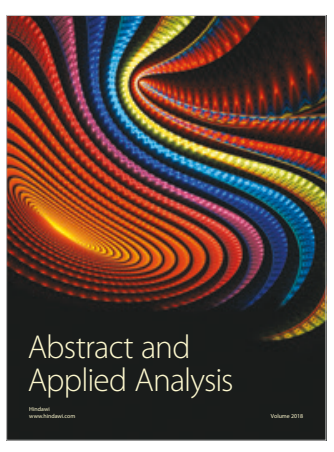

The Scientific

World Journal

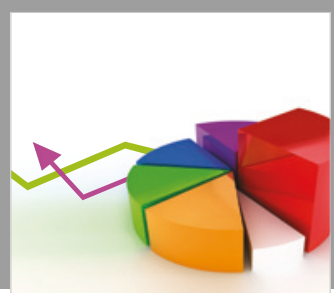

Journal of

Probability and Statistics
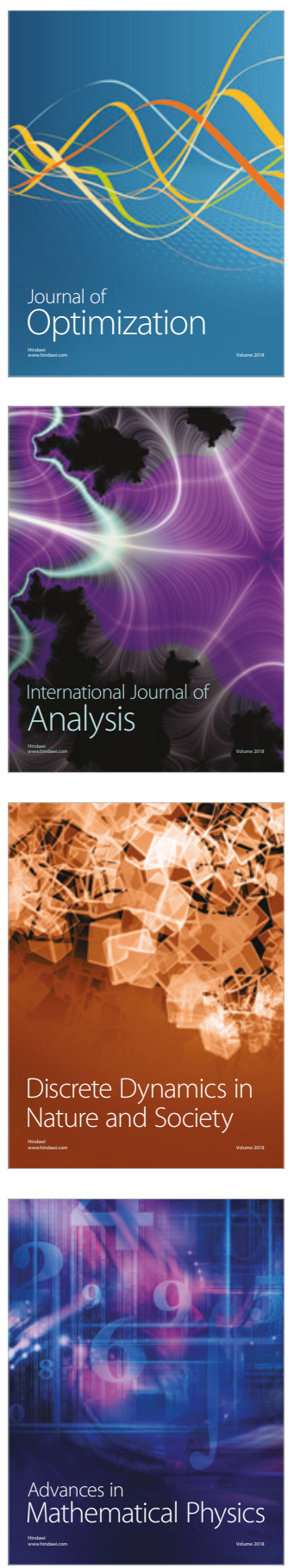